\title{
SEMILINEAR FRACTIONAL DIFFERENTIAL EQUATIONS: GLOBAL SOLUTIONS, CRITICAL NONLINEARITIES AND COMPARISON RESULTS
}

\author{
Bruno de Andrade - Alexandre N. Carvalho \\ Paulo M. Carvalho-Neto - Pedro Marín-Rubio
}

\begin{abstract}
In this work we study several questions concerning to abstract fractional Cauchy problems of order $\alpha \in(0,1)$. Concretely, we analyze the existence of local mild solutions for the problem, and its possible continuation to a maximal interval of existence. The case of critical nonlinearities and corresponding regular mild solutions is also studied. Finally, by establishing some general comparison results, we apply them to conclude the global well-posedness of a fractional partial differential equation coming from heat conduction theory.
\end{abstract}

2010 Mathematics Subject Classification. Primary: 34A08, 34G20; Secondary: 35K05, 35B09.

Key words and phrases. Fractional differential equations; semilinear equations; comparison results; critical nonlinearities.

The first named author partially supported by CNPQ 100994/2011-3 and 478053/2013-4, and CAPES BEX 5549/11-6.

Research of the second named author partially supported by CNPq 305447/2005-0 and 451761/2008-1, CAPES/DGU 267/2008 and FAPESP 2008/53094-4, Brazil.

The third named author partially supported by FAPESP - Brazil, grant \# 2008/58944-6, and CAPES - Brazil, grant \# BEX 5537/11-8.

The fourth named author partially supported by Ministerio de Educación-DGPU (Spain) project PHB2010-0002-PC, Ministerio de Ciencia e Innovación (Spain) project MTM201122411, and Junta de Andalucía grant P07-FQM-02468. 
B. De Andrade - A.N. Carvalho - P.M. Carvalho-Neto - P. Marín-Rubio

\section{Introduction and statement of the results}

Origins of fractional calculus go back to a question posed by Leibniz to L'Hôpital about the meaning of the derivative of order $1 / 2$. Since then this theory was developed and nowadays it has generated a considerable amount of bibliography, see for example the monographs [11], [13], [14], [17] and the references therein.

Naturally the interest in fractional differential equations was considerably raised over the years; among engineers and scientists this is due to their vast potential of applications in several applied problems, such as fluid flow, rheology, diffusive transport akin to diffusion, electric networks, probability and statistical distribution theory, see [4], [18]-[20], [25] among others.

From the mathematical point of view, we observe that fractional differential equations is a rich and complex area and hence there exists a great interest in developing the theoretical analysis and numerical methods for these equations, see [7], [8], [10], [12], [15], [16], [21], [22].

Motivated by this, we consider the abstract fractional Cauchy problem

$$
\left\{\begin{array}{l}
c D_{t}^{\alpha} u(t)=A u(t)+f(t, u(t)), \quad t>0, \\
u(0)=u_{0} \in X,
\end{array}\right.
$$

where $X$ is a Banach space, $\alpha \in(0,1), c D_{t}^{\alpha}$ is Caputo's fractional derivative and $A: D(A) \subset X \rightarrow X$ is a sectorial operator, i.e. a closed operator with dense domain for which there are $\phi \in(\pi / 2, \pi)$ and $N \geq 1$ such that

$$
\begin{gathered}
S_{\phi}:=\{\lambda \in \mathbb{C}:|\arg (\lambda)| \leq \phi, \lambda \neq 0\} \subset \rho(A), \\
\left\|(\lambda-A)^{-1}\right\| \leq \frac{N}{|\lambda|} \text { for all } \lambda \in S_{\phi} .
\end{gathered}
$$

In order to start our discussion, let us introduce some preliminaries. Consider $\left\{E_{\alpha}\left(t^{\alpha} A\right)\right\}_{t \geq 0}$ and $\left\{E_{\alpha, \alpha}\left(t^{\alpha} A\right)\right\}_{t \geq 0}$ the Mittag-Leffler families associated to $A$ (see next section for details). By a global mild solution to $(1.1)$ in $[0, \infty)$ we understand a continuous function $u:[0, \infty) \rightarrow X$ such that

$$
u(t)=E_{\alpha}\left(t^{\alpha} A\right) u_{0}+\int_{0}^{t}(t-s)^{\alpha-1} E_{\alpha, \alpha}\left((t-s)^{\alpha} A\right) f(s, u(s)) d s, \quad t \geq 0 .
$$

This concept is based upon the following (formal) construction. Suppose that $u:[0, \infty) \rightarrow X$ verifies (1.1). Then, applying the fractional integral operator $J_{t}^{\alpha}$ (see, e.g. [3], [12]-[14]) in both sides of the fractional differential equation (1.1), we obtain, for $t \geq 0$,

$$
\begin{aligned}
u(t) & =u(0)+J_{t}^{\alpha} A u(t)+J_{t}^{\alpha} f(t, u(t)) \\
& =u_{0}+\frac{1}{\Gamma(\alpha)} \int_{0}^{t}(t-s)^{\alpha-1} A u(s) d s+\frac{1}{\Gamma(\alpha)} \int_{0}^{t}(t-s)^{\alpha-1} f(s, u(s)) d s .
\end{aligned}
$$


Now, applying the Laplace transform $\mathcal{L}$ in both sides of the above equality we have

$\widehat{u}(\lambda)=\frac{u_{0}}{\lambda}+\frac{1}{\lambda^{\alpha}} A \widehat{u}(\lambda)+\frac{1}{\lambda^{\alpha}}(\widehat{f(u)})(\lambda) \Rightarrow \lambda^{\alpha} \widehat{u}(\lambda)=u_{0} \lambda^{\alpha-1}+A \widehat{u}(\lambda)+(\widehat{f(u)})(\lambda)$, where $\widehat{u}(\lambda)=\mathcal{L}\{u(t)\}(\lambda)$ and $\widehat{f(u)}(\lambda)=\mathcal{L}\{f(t, u(t))\}(\lambda)$. Taking now $\lambda^{\alpha} \in$ $\rho(A)$, it follows that

$$
\widehat{u}(\lambda)=\lambda^{\alpha-1}\left(\lambda^{\alpha}-A\right)^{-1} u_{0}+\left(\lambda^{\alpha}-A\right)^{-1}(\widehat{f(u)})(\lambda),
$$

and using the inverse Laplace transform, and Lemma 2.1 below, we deduce that

$$
u(t)=E_{\alpha}\left(t^{\alpha} A\right) u_{0}+\int_{0}^{t}(t-s)^{\alpha-1} E_{\alpha, \alpha}\left((t-s)^{\alpha} A\right) f(s, u(s)) d s, \quad t \geq 0 .
$$

Motivated by this discussion and by the previous related literature, we adopt the following concept for solution to the problem (1.1).

DeFinition 1.1. Let $\tau>0$.

(a) A function $u:[0, \tau] \rightarrow X$ is said to be a local mild solution to (1.1) in $[0, \tau]$ if $u \in C([0, \tau] ; X)$ and

$$
u(t)=E_{\alpha}\left(t^{\alpha} A\right) u_{0}+\int_{0}^{t}(t-s)^{\alpha-1} E_{\alpha, \alpha}\left((t-s)^{\alpha} A\right) f(s, u(s)) d s, \quad t \geq 0 .
$$

(b) A function $u:[0, \tau) \rightarrow X$ is said to be a local mild solution to (1.1) in $[0, \tau)$ if for any $\tau^{\prime} \in[0, \tau), u$ is a local mild solution to (1.1) in $\left[0, \tau^{\prime}\right]$.

Our main purpose in this work is to ensure sufficient conditions for existence and uniqueness of mild solution to (1.1) and to establish some comparison results for this solution in ordered Banach spaces. As can be observed in (1.2), the notion of mild solution to (1.1) is very close to generalized Mittag-Leffler type functions. For this, in Section 2, among other things, we study some properties of these functions. In particular, we study the behavior of the families $\left\{E_{\alpha}\left(t^{\alpha} A\right)\right\}_{t \geq 0}$ and $\left\{E_{\alpha, \alpha}\left(t^{\alpha} A\right)\right\}_{t \geq 0}$ on the fractional power spaces associated to the linear operator $A$ and we establish expressions for these families, similar to the second fundamental limit for semigroups (see Lemma 2.2 and Proposition 2.4 below).

Section 3 is devoted to study existence, uniqueness and continuation results to (1.1) when the nonlinear term is a locally Lipschitz continuous function $f:[0, \infty) \times X \rightarrow X$. In this direction we start with a proof of the following existence result.

TheOrem 1.2. Let $f:[0, \infty) \times X \rightarrow X$ be a continuous function, and locally Lipschitz in the second variable, uniformly with respect to the first variable, that $i s$, for each fixed $x \in X$, there exist an open ball $B_{x}$ and a constant $L=L\left(B_{x}\right) \geq 0$ such that

$$
\|f(t, z)-f(t, y)\| \leq L\|z-y\|
$$


442 B. de Andrade - A.N. Carvalho - P.M. Carvalho-Neto - P. Marín-Rubio

for all $z, y \in B_{x}$ and $t \in[0, \infty)$. Then, there exists $t_{0}>0$ such that (1.1) has a unique local mild solution in $\left[0, t_{0}\right]$.

In the remainder of Section 3 our goal is to ensure conditions for existence of global solutions of (1.1). For this purpose, we prove the following result on continuation of mild solutions.

Theorem 1.3. Let $f:[0, \infty) \times X \rightarrow X$ be as in Theorem 1.2. If $u:\left[0, t_{0}\right] \rightarrow X$ is a local mild solution to (1.1) in $\left[0, t_{0}\right]$, then there exists a unique continuation $u^{*}$ of $u$ in some interval $\left[0, t_{0}+\tau\right]$ with $\tau>0$.

We finish Section 3 with a result on global existence or non-continuation by blow-up. Namely, we have the following

Theorem 1.4. Let $f:[0, \infty) \times X \rightarrow X$ be a continuous function, locally Lipschitz in the second variable, uniformly with respect to the first variable, and bounded (i.e. it maps bounded sets onto bounded sets). Then the problem (1.1) has a global mild solution in $[0, \infty)$ or there exists $\omega \in(0, \infty)$ such that $u:[0, \omega) \rightarrow X$ is a local mild solution in $[0, \omega)$ and $u$ does not have a continuation. Moreover, if $u$ is the local mild solution to (1.1) in $[0, \omega)$, then $\limsup _{t \rightarrow \omega^{-}}\|u(t)\|=\infty$.

We observe that the assumption on the nonlinear term $f$ is rather general but it does not allow to treat problems where the nonlinearity has critical growth. In Section 4 we treat the critical case, proving existence of $\varepsilon$-regular mild solution to $(1.1)$.

In order to state this result, let us introduce some notation. For $\beta \geq 0$, let $X^{\beta}$ be the fractional power spaces associated to the operator $A$. In this work we will use the following concepts.

Definition 1.5. A continuous function $u:[0, \tau] \rightarrow X^{1}$ is called an $\varepsilon$-regular mild solution to (1.1) if $u \in C\left((0, \tau] ; X^{1+\varepsilon}\right)$ and verifies (1.2).

Definition 1.6. For $\varepsilon>0$ we say that a map $g$ is an $\varepsilon$-regular map relative to the pair $\left(X^{1}, X^{0}\right)$ if there exist $\rho>1, \gamma(\varepsilon)$ with $\rho \varepsilon \leq \gamma(\varepsilon)<1$, and a positive constant $c$, such that $g: X^{1+\varepsilon} \rightarrow X^{\gamma(\varepsilon)}$ and, for all $x, y \in X^{1+\varepsilon}$,

$$
\|g(x)-g(y)\|_{X^{\gamma(\varepsilon)}} \leq c\left(1+\|x\|_{X^{1+\varepsilon}}^{\rho-1}+\|y\|_{X^{1+\varepsilon}}^{\rho-1}\right)\|x-y\|_{X^{1+\varepsilon}} .
$$

We recall from [2] the following classification for a map $f$ which is $\varepsilon$-regular, for $\varepsilon \in I$, relative to the pair $\left(X^{1}, X^{0}\right)$.

- If $I=\left[0, \varepsilon_{1}\right]$ for some $\varepsilon_{1}>0$ and $\gamma(0)>0$, we say that $f$ is a subcritical map relative to $\left(X^{1}, X^{0}\right)$.

- If $I=\left[0, \varepsilon_{1}\right]$ for some $\varepsilon_{1}>0$ with $\gamma(\varepsilon)=\rho \varepsilon, \varepsilon \in I$, and if $f$ is not subcritical, then we say that $f$ is a critical map relative to $\left(X^{1}, X^{0}\right)$. 
- If $I=\left(0, \varepsilon_{1}\right]$ for some $\varepsilon_{1}>0$ with $\gamma(\varepsilon)=\rho \varepsilon, \varepsilon \in I$, and $f$ is not subcritical or critical, then we say that $f$ is a double-critical map relative to $\left(X^{1}, X^{0}\right)$.

- If $I=\left[\varepsilon_{0}, \varepsilon_{1}\right]$ for some $\varepsilon_{1}>\varepsilon_{0}>0$ with $\gamma\left(\varepsilon_{0}\right)>\rho \varepsilon_{0}$ and $f$ is not subcritical, critical or double critical, then we say that $f$ is an ultrasubcritical map relative to $\left(X^{1}, X^{0}\right)$.

- If $I=\left[\varepsilon_{0}, \varepsilon_{1}\right]$ for some $\varepsilon_{1}>\varepsilon_{0}>0$ with $\gamma(\varepsilon)=\rho \varepsilon, \varepsilon \in I$, and if $f$ is not subcritical, critical, double critical or ultra-subcritical, then we say that $f$ is an ultra-critical map relative to $\left(X^{1}, X^{0}\right)$.

Next theorem is the main result of Section 4 (the class $\mathcal{F}(\varepsilon, \rho, \gamma(\varepsilon), c, \nu(\cdot), \xi)$ is specified).

Theorem 1.7. Let $f \in \mathcal{F}(\varepsilon, \rho, \gamma(\varepsilon), c, \nu(\cdot)$, $\xi)$. If $v_{0} \in X^{1}$, there exist positive values $r$ and $\tau_{0}$ such that for any $u_{0} \in B_{X^{1}}\left(v_{0}, r\right)$ there exists a continuous function $u\left(\cdot, u_{0}\right):\left[0, \tau_{0}\right] \rightarrow X^{1}$ with $u(0)=u_{0}$, which is an $\varepsilon$-regular mild solution to the problem

$$
\left\{\begin{array}{l}
c D_{t}^{\alpha} u=A u+f(t, u(t)), \quad t>0, \alpha \in(0,1), \\
u(0)=u_{0} .
\end{array}\right.
$$

This solution satisfies

$$
\begin{array}{ll}
u \in C\left(\left(0, \tau_{0}\right] ; X^{1+\theta}\right), & 0 \leq \theta<\gamma(\varepsilon), \\
\lim _{t \rightarrow 0^{+}} t^{\alpha \theta}\left\|u\left(t, u_{0}\right)\right\|_{X^{1+\theta}}=0, & 0<\theta<\gamma(\varepsilon) .
\end{array}
$$

Moreover, for each $\theta_{0}<\gamma(\varepsilon)+\varepsilon-\rho \varepsilon$ there exists a constant $C>0$ such that if $u_{0}, w_{0} \in B_{X^{1}}\left(v_{0}, r\right)$, then

$$
t^{\alpha \theta}\left\|u\left(t, u_{0}\right)-u\left(t, w_{0}\right)\right\|_{X^{1+\theta}} \leq C\left\|u_{0}-w_{0}\right\|_{X^{1}} \quad \text { for all } t \in\left[0, \tau_{0}\right], 0 \leq \theta \leq \theta_{0} .
$$

In particular we obtain an existence theorem in $X^{1}$ without the nonlinearity being defined on $X^{1}$. The main motivation to consider situations as in Theorem 1.7 is the fact that if the only requirement on the nonlinear term is that $f: X^{1} \rightarrow X^{0}$ be locally Lipschitz, we cannot ensure that problem (1.1) is wellposed in an $\varepsilon$-regular sense. For example, taking $f(u)=-2 A u$, which satisfies $f: X^{1} \rightarrow X^{0}$ and is globally Lipschitz, we will have $c D_{t}^{\alpha} u=-A u$, which is not locally well-posed, in general. Hence, some extra conditions should be imposed on $f$ to guarantee the existence of solutions of the above problem.

As application we consider our abstract result in the framework of fractional heat equations. 
444 B. de Andrade - A.N. Carvalho - P.M. Carvalho-Neto - P. Marín-Rubio

EXAMPLE 1.8. Let $\Omega \subset \mathbb{R}^{N}$ be a bounded domain with smooth boundary. We will treat the fractional equation (with $\alpha \in(0,1)$ )

$$
\begin{cases}\partial_{t}^{\alpha} u=\Delta u+u|u|^{\rho-1} & \text { in } \Omega, \\ u=0 & \text { on } \partial \Omega,\end{cases}
$$

in the $L^{q}$ theory, $1<q<\infty$ and $q=N(\rho-1) / 2$. Observe that this equation for the case $\alpha=1$ is well-known (e.g. cf. [2], [5], [23], [24]).

Let $L=\Delta$ with Dirichlet boundary conditions in $\Omega$. Then, $L$ can be seen as an unbounded operator in $E_{q}^{0}=L^{q}(\Omega)$ with domain

$$
D(A):=W^{2, q}(\Omega) \cap W_{0}^{1, q}(\Omega) .
$$

It is well-known that the scale of fractional power spaces $\left\{E_{q}^{\alpha}\right\}_{\varepsilon \in \mathbb{R}}$ associated to $L$ verifies (see [1], [2])

$$
\begin{aligned}
E_{q}^{\alpha} \hookrightarrow H_{q}^{2 \alpha}(\Omega), & \alpha \geq 0,1<q<\infty, \\
E_{q}^{-\alpha} \hookrightarrow\left(E_{q^{\prime}}^{\alpha}\right)^{\prime}, & \alpha \geq 0,1<q<\infty, q^{\prime}=\frac{q}{q-1} .
\end{aligned}
$$

Therefore, we have

$$
\begin{aligned}
& E_{q}^{\alpha} \hookrightarrow L^{r}(\Omega) \quad \text { for } r \leq \frac{N q}{N-2 q \alpha}, 0 \leq \alpha \leq \frac{N}{2 q}, \\
& E_{q}^{0}=L^{q}(\Omega), \\
& E_{q}^{\alpha} \hookleftarrow L^{r}(\Omega) \quad \text { for } r \geq \frac{N}{N-2 q \alpha}, \quad-\frac{N}{2 q^{\prime}} \leq \alpha \leq 0 .
\end{aligned}
$$

Let $L_{\alpha}$ be the realization of $L$ in $E_{q}^{\alpha}$. Then we have that $L_{\alpha}$ is an isometry from $E_{q}^{\alpha+1}$ into $E_{q}^{\alpha}$ and

$$
L_{\alpha}: D\left(L_{\alpha}\right)=E_{q}^{\alpha+1} \subset E_{q}^{\alpha} \rightarrow E_{q}^{\alpha}
$$

is a sectorial operator. Denote $X_{q}^{\alpha}:=E_{q}^{\alpha-1}, \alpha \in \mathbb{R}$. The fractional power spaces associated to $L_{-1}: X_{q}^{1} \subset X_{q}^{0} \rightarrow X_{q}^{0}$ satisfy

$$
\begin{aligned}
& X_{q}^{\alpha} \hookrightarrow L^{r}(\Omega) \quad \text { for } r \leq \frac{N q}{N+2 q-2 q \alpha}, 1 \leq \alpha<\frac{2 q+N}{2 q}, \\
& X_{q}^{1}=L^{q}(\Omega), \\
& X_{q}^{\alpha} \hookleftarrow L^{r}(\Omega) \quad \text { for } r \geq \frac{N q}{N+2 q-2 q \alpha}, \quad \frac{2 q^{\prime}-N}{2 q^{\prime}}<\alpha \leq 1 .
\end{aligned}
$$

Following [2, Lemma 8] and with the above constructions, we may classify the nonlinear term of (1.4) in the following way.

- If $\rho>N /(N-2)$, then $f$ is an $\varepsilon$-regular map relative to $\left(X_{q}^{1}, X_{q}^{0}\right)$ for $0 \leq \varepsilon<N /(N+2 q)$ and $\gamma(\varepsilon)=\rho \varepsilon$. Therefore $f$ is a critical map.

- If $\rho=N /(N-2)$, then $f$ is an $\varepsilon$-regular map relative to $\left(X_{q}^{1}, X_{q}^{0}\right)$ for $0<\varepsilon<N /(N+2 q)$ and $\gamma(\varepsilon)=\rho \varepsilon$. Therefore $f$ is a double-critical map. 
- If $(N+2) / N<\rho<N /(N-2)$, then $f$ is an $\varepsilon$-regular map relative to $\left(X_{q}^{1}, X_{q}^{0}\right)$ for $0<\varepsilon_{0}(\rho)<\varepsilon<1 / \rho$, with

$$
\varepsilon_{0}(\rho)=\frac{1}{\rho}\left(1-\frac{N}{2}\left(1-\frac{2}{N(\rho-1)}\right)\right)>0,
$$

and $\gamma(\varepsilon)=\rho \varepsilon$. Therefore $f$ is an ultra-critical map.

Applying Theorem 1.7, for each $u_{0} \in L^{q}(\Omega)$ we ensure the existence of an $\varepsilon$-regular solution to the above problem, starting at $u_{0}$, for any $\varepsilon \in\left(\varepsilon_{0}(\rho), N /(N+2 q)\right)$. Furthermore, for any $0<\theta<\gamma(N /(N+2))=1$ this solution verifies

$$
\begin{gathered}
t^{\alpha \theta}\|u(t)\|_{X^{1+\theta}} \rightarrow 0 \quad \text { as } t \rightarrow 0^{+}, \\
t^{\alpha \theta}\left\|u\left(t, u_{0}\right)-u\left(t, v_{0}\right)\right\|_{X^{1+\theta}} \leq C\left\|u_{0}-v_{0}\right\|_{L^{q}}, \quad 0<t<\tau\left(u_{0}, v_{0}\right) .
\end{gathered}
$$

In Section 5 we consider the problem of comparison and positive solutions. We start this with the linear version of (1.1); particularly, we establish the equivalence between the positivity of the families $\left\{E_{\alpha}\left(t^{\alpha} A\right)\right\}_{t \geq 0}$ and $\left\{E_{\alpha, \alpha}\left(t^{\alpha} A\right)\right\}_{t \geq 0}$ with the positivity of the function $\lambda \mapsto\left(\lambda^{\alpha}-A\right)^{-1}$ (see Propositions 5.1 and 5.3 below). With respect to the semilinear problem (1.1), we establish the following result on positive solutions.

Theorem 1.9. Let $\left(X, \leq_{X}\right)$ be an ordered Banach space and suppose that the families $\left\{E_{\alpha}\left(t^{\alpha} A\right)\right\}_{t \geq 0}$ and $\left\{E_{\alpha, \alpha}\left(t^{\alpha} A\right)\right\}_{t \geq 0}$ are increasing. If $0 \leq_{X} f(t, x)$ for almost every $t \in\left[0, t_{0}\right)$ and for all $x \in X$ with $0 \leq_{X} x$, then $0 \leq_{X} u_{0}$ implies that the local mild solution $u_{f}\left(t, u_{0}\right)$ is positive, i.e. $0 \leq_{X} u_{f}\left(t, u_{0}\right)$ for all $t \in\left[0, t_{0}\right]$.

This allows us to conclude the following comparison result.

Theorem 1.10. Let $\left(X, \leq_{X}\right)$ be an ordered Banach space and suppose that the families $\left\{E_{\alpha}\left(t^{\alpha} A\right)\right\}_{t \geq 0}$ and $\left\{E_{\alpha, \alpha}\left(t^{\alpha} A\right)\right\}_{t \geq 0}$ are increasing.

(a) Let $u_{0}, u_{1} \in X$ be given, and assume that there exist $t_{0}, t_{1} \in[0, \infty)$ such that $\left\{u_{f}\left(t, u_{i}\right)\right\}_{i=0,1}$ are local mild solutions in $\left[0, t_{i}\right], i \in\{0,1\}$, to

$$
\left\{\begin{array}{l}
c D_{t}^{\alpha} u(t)=A u(t)+f(t, u(t)), \quad t>0, \\
u(0)=u_{i} \in X .
\end{array}\right.
$$

Then, if $t^{*}=\min \left\{t_{0}, t_{1}\right\}, f(t, \cdot)$ is increasing for almost every $t \in\left[0, t^{*}\right)$, and $u_{1} \leq_{X} u_{0}$, it holds that $u_{f}\left(t, u_{1}\right) \leq_{X} u_{f}\left(t, u_{0}\right)$ for all $t \in\left[0, t^{*}\right]$.

(b) Consider functions $f_{0}$ and $f_{1}$, and $u_{0} \in X$, and assume that there exist $t_{0}, t_{1} \in[0, \infty)$ such that $\left\{u_{f_{i}}\left(t, u_{0}\right)\right\}_{i=0,1}$ are local mild solutions in $\left[0, t_{i}\right]$, $i \in\{0,1\}$, to

$$
\left\{\begin{array}{l}
c D_{t}^{\alpha} u(t)=A u(t)+f_{i}(t, u(t)), \quad t>0 \\
u(0)=u_{0} \in X
\end{array}\right.
$$


446 B. de Andrade - A.N. Carvalho - P.M. Carvalho-Neto - P. Marín-Rubio

Then, if $t^{*}=\min \left\{t_{0}, t_{1}\right\}$ and $f_{0}(t, x) \leq_{X} f_{1}(t, x)$ for almost every $t \in$ $\left[0, t^{*}\right]$ and for all $x \in X$, it holds that $u_{f_{0}}\left(t, u_{0}\right) \leq_{X} u_{f_{1}}\left(t, u_{0}\right)$ for all $t \in\left[0, t^{*}\right]$.

(c) Consider functions $f_{0}$ and $f_{1}$, and $u_{0}, u_{1} \in X$, and assume that there exist $t_{0}, t_{1} \in[0, \infty)$ such that $\left\{u_{f_{i}}\left(t, u_{i}\right)\right\}_{i=0,1}$ are local mild solutions in $\left[0, t_{i}\right], i \in\{0,1\}$, to

$$
\left\{\begin{array}{l}
c D_{t}^{\alpha} u(t)=A u(t)+f_{i}(t, u(t)), \quad t>0, \\
u(0)=u_{i} \in X
\end{array}\right.
$$

Then, if $t^{*}=\min \left\{t_{0}, t_{1}\right\}$, and $x \leq_{X} y$ imply $f_{0}(t, x) \leq_{X} f_{1}(t, y)$ for almost every $t \in\left[0, t^{*}\right]$ and $u_{0} \leq_{X} u_{1}$, it holds that $u_{f_{0}}\left(t, u_{0}\right) \leq_{X} u_{f_{1}}\left(t, u_{1}\right)$ for all $t \in\left[0, t^{*}\right]$.

Next result uses the comparison of solutions to establish the existence of a unique mild solution in a certain interval. As a consequence, when the solutions of the auxiliary problems are globally defined in time, from the continuation results we obtained previously, we will conclude the existence of a unique global mild solution (see Example 1.12 below).

Corollary 1.11. Let $\left(X, \leq_{X}\right)$ be an ordered Banach space and $C>0$ such that

$$
x \leq_{X} y \leq_{X} z \Rightarrow\|y\|_{X} \leq C\left(\|x\|_{X}+\|z\|_{X}\right) \quad \text { for all } x, y, z \in X .
$$

Suppose that the families $\left\{E_{\alpha}\left(t^{\alpha} A\right)\right\}_{t \geq 0}$ and $\left\{E_{\alpha, \alpha}\left(t^{\alpha} A\right)\right\}_{t \geq 0}$ are increasing. Let $u_{0} \in X$ and consider $f_{1}, f_{2}$ and $g:[0, \infty) \times X \rightarrow X$ as in Theorem 1.2, and that $g$ is bounded. Moreover, assume that $f_{1}(t, x) \leq_{X} g(t, x) \leq_{X} f_{2}(t, x)$ for almost every $t \geq 0$ and for all $x \in X$. Suppose that the problems

$$
\left\{\begin{array}{l}
c D_{t}^{\alpha} u(t)=A u(t)+f_{i}(t, u(t)), \quad t>0 \\
u(0)=u_{0} \in X
\end{array}\right.
$$

for $i=\{1,2\}$ have unique local mild solutions in $\left[0, t^{*}\right]$ for some $t^{*}>0$. Then, the problem

$$
\left\{\begin{array}{l}
c D_{t}^{\alpha} u(t)=A u(t)+g(t, u(t)), \quad t>0, \\
u(0)=u_{0} \in X .
\end{array}\right.
$$

has a unique local mild solution in $\left[0, t^{*}\right]$.

Finally, as an application of this last result (and some of Section 3) we ensure existence of global mild solution for the following fractional partial differential equation coming from heat conduction theory: 
EXAmple 1.12. Let $\Omega \subset \mathbb{R}^{N}, N \in \mathbb{N}$, be a bounded open subset with smooth boundary $\partial \Omega$. Consider the problem

$$
\begin{cases}c \partial_{t}^{\alpha} u(t, x)=D \Delta u(t, x)+F(u(t, x)), & t>0, x \in \Omega, \\ \partial u / \partial \vec{v}(t, x)=0, & t>0, x \in \partial \Omega, \\ u(0, x)=u_{0}(x), & x \in \Omega .\end{cases}
$$

where $c \partial_{t}^{\alpha}$ is Caputo's fractional derivative, $u=\left(u_{1}, \ldots, u_{n}\right)^{\top}, n \geq 1$,

$$
\frac{\partial u}{\partial \vec{v}}=\left(\left\langle\nabla u_{1}, \vec{v}\right\rangle, \ldots,\left\langle\nabla u_{n}, \vec{v}\right\rangle\right)^{\top}
$$

where $\vec{v}$ is the outward normal vector, and $D$ is the diagonal matrix

$$
D=\left[\begin{array}{ccccc}
d_{1} & 0 & 0 & \ldots & 0 \\
0 & d_{2} & 0 & \ldots & 0 \\
0 & 0 & d_{3} & \ldots & 0 \\
\vdots & \vdots & \vdots & \ddots & \vdots \\
0 & 0 & 0 & \ldots & d_{n}
\end{array}\right]_{n \times n}
$$

with $d_{i}>0$, for all $i \in\{0,1, \ldots, n\}$. The nonlinearity

$$
F=\left(F_{1}, \ldots, F_{n}\right): \mathbb{R}^{n} \rightarrow \mathbb{R}^{n}
$$

is assumed to be locally Lipschitz.

To treat this problem we set $X=\left(L^{q}(\Omega)\right)^{n}$, for $1<q<\infty$, with the usual order $\leq_{X}$ and we consider $A_{D}=\operatorname{diag}\left(A_{1}, \ldots A_{n}\right)$, where for each $i \in\{0, \ldots, n\}$ we define $A_{i}: D\left(A_{i}\right) \subset L^{q}(\Omega) \rightarrow L^{q}(\Omega)$ by

$$
\begin{aligned}
D\left(A_{i}\right) & =\left\{\phi \in W^{2, q}(\Omega): \frac{\partial \phi}{\partial \vec{v}}=0 \text { on } \partial \Omega\right\}=: W_{\mathcal{N}}^{2, q}(\Omega) ; \\
A_{i} \phi & =-d_{i} \Delta \phi
\end{aligned}
$$

and suppose that $\inf \left\{\operatorname{Re}\left(\sigma\left(A_{D}\right)\right)\right\}=\widetilde{d}_{1}<0$. In [6, Proposition 2.8] it was shown that this operator is sectorial and has positive resolvent. Then, rewriting (1.5) in the abstract form

$$
\left\{\begin{array}{l}
c D_{t}^{\alpha} u(t)=-A_{D} u(t)+F(u(t)), \quad t>0, \\
u(0)=u_{0} \in\left(L^{q}(\Omega)\right)^{n}
\end{array}\right.
$$

we may ensure, by Theorem 1.2, existence and uniqueness of a local mild solution $u$. If there exist constants $c_{0} \in\left[0,-\widetilde{d}_{1}\right)$ and $c_{1} \geq 0$ such that

$$
-c_{0}\left|u_{i}\right|-c_{1} \leq F_{i}(u) \leq c_{0}\left|u_{i}\right|+c_{1} \quad \text { for all } i \in\{1, \ldots, n\},
$$

then problem (1.5) has a global mild solution. Indeed, we conclude that

$$
-c_{0}\|u\|-C_{1} \leq_{X} F(u) \leq_{X} c_{0}\|u\| \|+C_{1} \text { for all } u \in\left(L^{q}(\Omega)\right)^{n},
$$

where $\||u|\|:=\left(\left|u_{1}\right|, \ldots,\left|u_{n}\right|\right)$ and $C_{1}=\left(c_{1}, \ldots, c_{1}\right)$. 
Since the problems

$$
\left\{\begin{array}{l}
c D_{t}^{\alpha} u(t)=-A_{D} u(t)-c_{0}\|u(t)\|-C_{1}, \quad t>0, \\
u(0)=u_{0} \in\left(L^{q}(\Omega)\right)^{n},
\end{array}\right.
$$

and

$$
\left\{\begin{array}{l}
c D_{t}^{\alpha} u(t)=-A_{D} u(t)+c_{0}\|u(t)\|+C_{1}, \quad t>0, \\
u(0)=u_{0} \in\left(L^{q}(\Omega)\right)^{n},
\end{array}\right.
$$

have global mild solutions (by using Theorem 1.4), we conclude by Corollary 1.11 and Theorems 1.3 and 1.4 that (1.5) possesses a unique global mild solution.

\section{Preliminary results}

Let $(X,\|\cdot\|)$ be a complex Banach space. As usual, for a linear operator $A$, we denote by $D(A)$ the domain of $A$, by $\sigma(A)$ its spectrum, while $\rho(A):=\mathbb{C}-\sigma(A)$ is the resolvent set of $A$. Moreover, we denote by $\mathcal{L}(Y, Z)$ the space of all bounded linear operators between two normed spaces $Y$ and $Z$ with the operator norm $\|\cdot\|_{\mathcal{L}(Y, Z)}$. We abbreviate this notation to $\mathcal{L}(Y)$ when $Y=Z$. When no confusion arise, we write $\|T\|_{\mathcal{L}(Y)}$ as $\|T\|$ for every $T \in \mathcal{L}(Y)$. Finally, for $\beta \geq 0$ we denote by $X^{\beta}$ the fractional power spaces associated to the operator $A$, being $X^{0}=X$ and $X^{1}=D(A)$.

The aim of this section is to present the basic tools we will use in this manuscript. Our results include the classical theory of the Mittag-Leffler function that is closely related to the solutions of the fractional differential equations.

2.1. Mittag-Leffler functions and sectorial operators. We start with a generalization of the Cauchy representation for semigroups associated to sectorial operators. To this end we recall that given $\varepsilon>0$ and $\theta \in(\pi / 2, \pi)$, the Hankel path $H a=H a(\varepsilon, \theta)$ is the path given by $H a=H a_{1}+H a_{2}-H a_{3}$, where $H a_{i}$ are such that

$$
\begin{aligned}
& H a_{1}:=\left\{t e^{i \theta}: t \in[\varepsilon, \infty)\right\}, \\
& H a_{2}:=\left\{\varepsilon e^{i t}: t \in[-\theta, \theta]\right\}, \\
& H a_{3}:=\left\{t e^{-i \theta}: t \in[\varepsilon, \infty)\right\} .
\end{aligned}
$$

Lemma 2.1. Consider $\alpha \in(0,1)$ and suppose that $A: D(A) \subset X \rightarrow X$ is a sectorial operator. Then, the functions

$$
\begin{aligned}
E_{\alpha}\left(t^{\alpha} A\right) & :=\frac{1}{2 \pi i} \int_{H a} e^{\lambda t} \lambda^{\alpha-1}\left(\lambda^{\alpha}-A\right)^{-1} d \lambda, & t \geq 0, \\
E_{\alpha, \alpha}\left(t^{\alpha} A\right) & :=\frac{t^{1-\alpha}}{2 \pi i} \int_{H a} e^{\lambda t}\left(\lambda^{\alpha}-A\right)^{-1} d \lambda, & t \geq 0
\end{aligned}
$$


(where Ha is given by (2.1)) are well defined. Furthermore, there exists a constant $M>0$ such that

$$
\left\|E_{\alpha}\left(t^{\alpha} A\right)\right\| \leq M \quad \text { and } \quad\left\|E_{\alpha, \alpha}\left(t^{\alpha} A\right)\right\| \leq M \quad \text { for all } t \geq 0 .
$$

Proof. Let $\phi \in(\pi / 2, \pi), S_{\phi}$ be the sector associated with the sectorial operator $A$ and choose arbitrary values $\varepsilon>0$ and $\theta \in(\pi / 2, \phi]$. We will estimate the function $\left\|E_{\alpha}\left(t^{\alpha} A\right)\right\|$ on each $H a_{i}$, according to definition (2.1), for any $t>0$. Just observe that for each fixed $t \neq 0$, if we assume that $\varepsilon=1 / t$, then

- On $H a_{1}$, it holds that

$$
\begin{aligned}
& \left\|\frac{1}{2 \pi i} \int_{H a_{1}} e^{\lambda t} \lambda^{\alpha-1}\left(\lambda^{\alpha}-A\right)^{-1} d \lambda\right\| \\
& \leq \frac{1}{2 \pi}\left\|\int_{\varepsilon}^{\infty} e^{t s e^{i \theta}}\left(s e^{i \theta}\right)^{\alpha-1}\left(\left(s e^{i \theta}\right)^{\alpha}-A\right)^{-1} e^{i \theta} d s\right\|
\end{aligned}
$$

and using that if $\lambda=s e^{i \theta} \in H a(\varepsilon, \theta) \subset S_{\phi}$, then $\lambda^{\alpha} \in S_{\phi}$, we obtain by the sectorial property that

$$
\begin{aligned}
\left\|\frac{1}{2 \pi i} \int_{H a_{1}} e^{\lambda t} \lambda^{\alpha-1}\left(\lambda^{\alpha}-A\right)^{-1} d \lambda\right\| & \leq \frac{N}{2 \pi} \int_{\varepsilon}^{\infty} e^{t s \cos (\theta)}\left|\left(s e^{i \theta}\right)\right|^{-1} d s \\
& \leq \frac{N}{2 \pi \varepsilon} \int_{\varepsilon}^{\infty} e^{t s \cos (\theta)} d s=\frac{N e^{\cos (\theta)}}{-2 \pi \cos (\theta)}
\end{aligned}
$$

- On $H a_{2}$, we have that

$$
\begin{gathered}
\left\|\frac{1}{2 \pi i} \int_{H_{2}} e^{\lambda t} \lambda^{\alpha-1}\left(\lambda^{\alpha}-A\right)^{-1} d \lambda\right\| \\
=\frac{1}{2 \pi}\left\|\int_{-\theta}^{\theta} e^{t \varepsilon e^{i s}}\left(\varepsilon e^{i s}\right)^{\alpha-1}\left(\left(\varepsilon e^{i s}\right)^{\alpha}-A\right)^{-1} i \varepsilon e^{i s} d s\right\| \\
\leq \frac{N}{2 \pi} \int_{-\theta}^{\theta} e^{t \varepsilon \cos (s)} d s \leq \frac{\theta N e}{\pi} .
\end{gathered}
$$

- On $\mathrm{Ha}_{3}$ we proceed in the same way as in $H a_{1}$.

Taking $M$ as the maximum over all the bounds obtained above, we deduce that $\left\|E_{\alpha}\left(A t^{\alpha}\right)\right\|$ is well defined for each $t \geq 0$.

Now we shall seek a uniform bound for the function $\left\|E_{\alpha}\left(A t^{\alpha}\right)\right\|$ for all $t \geq 0$. For this, just observe that if $\varepsilon<\varepsilon^{\prime}$ and $\pi / 2<\theta^{\prime}<\theta<\phi$ and we take $H a=$ $H a(\varepsilon, \theta)$ and $H a^{\prime}=H a\left(\varepsilon^{\prime}, \theta^{\prime}\right)$, we obtain the equality

$\frac{1}{2 \pi i} \int_{H a} e^{\lambda t} \lambda^{\alpha-1}\left(\lambda^{\alpha}-A\right)^{-1} d \lambda=\frac{1}{2 \pi i} \int_{H a^{\prime}} e^{\lambda t} \lambda^{\alpha-1}\left(\lambda^{\alpha}-A\right)^{-1} d \lambda \quad$ for all $t \geq 0$.

This is enough to justify that the estimate obtained before is uniform on $t>0$. To verify the boundedness for $t=0$, we observe that making a change of variables

$$
E_{\alpha}\left(t^{\alpha} A\right)=\frac{1}{2 \pi i} \int_{H a} e^{\lambda} \lambda^{\alpha-1}\left(\lambda^{\alpha}-A t^{\alpha}\right)^{-1} d \lambda, \quad t \geq 0,
$$


450 B. De Andrade - A.N. Carvalho - P.M. Carvalho-Neto - P. Marín-Rubio

and therefore, using the dominated convergence theorem, we conclude that $E_{\alpha}\left(0^{\alpha} A\right)$ is the identity operator.

A similar procedure proves that $\left\|E_{\alpha, \alpha}\left(A t^{\alpha}\right)\right\| \leq M$ for all $t \in[0, \infty)$.

Next result give us information on the Mittag-Leffler families in the fractional power spaces $X^{\beta}, \beta \geq 0$, associated to the sectorial operator $A$.

Lemma 2.2. Consider $\alpha \in(0,1), 0 \leq \beta \leq 1$, and suppose that $A: D(A) \subset$ $X^{0} \rightarrow X^{0}$ is a sectorial operator. Then, there exists a constant $M>0$ such that

$$
\left\|E_{\alpha}\left(t^{\alpha} A\right) x\right\|_{X^{\beta}} \leq M t^{-\alpha \beta}\|x\|_{X^{0}}
$$

and

$$
\left\|t^{\alpha-1} E_{\alpha, \alpha}\left(t^{\alpha} A\right) x\right\|_{X^{\beta}} \leq M t^{\alpha(1-\beta)-1}\|x\|_{X^{0}}
$$

for all $t>0$.

Proof. We have that

$$
\begin{aligned}
\left\|E_{\alpha}\left(t^{\alpha} A\right) x\right\|_{X^{\beta}} & =\left\|\frac{1}{2 \pi i} \int_{H a} e^{\lambda t} \lambda^{\alpha-1} A^{\beta}\left(\lambda^{\alpha}-A\right)^{-1} x d \lambda\right\|_{X^{0}} \\
& \leq \frac{t^{-\alpha}}{2 \pi}\left\|\int_{H a} e^{\lambda} \lambda^{\alpha-1} A^{\beta}\left((\lambda / t)^{\alpha}-A\right)^{-1} x d \lambda\right\|_{X^{0}} \\
& \leq t^{-\alpha \beta}\left(\frac{C}{2 \pi} \int_{H a}\left|e^{\lambda} \lambda^{\alpha \beta-1}\right| d|\lambda|\right)\|x\|_{X^{0}} .
\end{aligned}
$$

Hence, it is sufficient to choose $M>0$ such that

$$
\frac{C}{2 \pi} \int_{H a}\left|e^{\lambda} \lambda^{\alpha \beta-1}\right| d|\lambda| \leq M
$$

By a similar procedure one may prove the second estimate.

Our goal now is to establish an expression for Mittag-Leffler functions associated to sectorial operators similar to the second fundamental limit for semigroups. For this, we recall the Post-Widder inversion formula, which can be found in [26]. This expression will be very useful to our following results.

Lemma 2.3 (Post-Widder). Let $u:[0, \infty) \rightarrow X$ be a continuous function such that $u(t)=O(\exp (\omega t))$ as $t \rightarrow \infty$ for some $\omega \in \mathbb{R}$ and let $\widehat{u}$ be the Laplace transform of $u$. Then

$$
u(t)=\lim _{n \rightarrow \infty} \frac{(-1)^{n}}{n !}\left(\frac{n}{t}\right)^{n+1}\left(\frac{d^{n}}{d \lambda^{n}} \widehat{u}\right)\left(\frac{n}{t}\right)
$$

uniformly on compact sets of $(0, \infty)$. 
Proposition 2.4. Consider $A: D(A) \subset X \rightarrow X$ a sectorial operator and $\alpha \in(0,1)$. Let $\left\{E_{\alpha}\left(t^{\alpha} A\right)\right\}_{t \geq 0}$ and $\left\{E_{\alpha, \alpha}\left(t^{\alpha} A\right)\right\}_{t \geq 0}$ be the Mittag-Leffler families associated to $A$. Then, for each $x \in X$ we have

$$
E_{\alpha}\left(t^{\alpha} A\right) x=\lim _{n \rightarrow \infty} \frac{1}{n !} \sum_{k=1}^{n+1} b_{k, n+1}^{\alpha}\left(\left(\frac{n}{t}\right)^{\alpha}\left(\left(\frac{n}{t}\right)^{\alpha}-A\right)^{-1}\right)^{k} x
$$

and

$$
t^{\alpha-1} E_{\alpha, \alpha}\left(t^{\alpha} A\right) u_{0}=\lim _{n \rightarrow \infty} \frac{1}{n !} \sum_{k=1}^{n} \alpha k b_{k, n}^{\alpha}\left(\frac{n}{t}\right)^{\alpha k+1}\left(\left(\frac{n}{t}\right)^{\alpha}-A\right)^{-(k+1)} x,
$$

uniformly on compact sets of $(0, \infty)$, where the positive real numbers $b_{k, n}^{\alpha}$ are defined by

$$
\begin{cases}b_{1,1}^{\alpha}=1, & \\ b_{k, n}^{\alpha}=(n-1-\alpha k) b_{k, n-1}^{\alpha}+\alpha(k-1) b_{k-1, n-1}^{\alpha}, & \text { if } 1 \leq k \leq n \\ b_{k, n}^{\alpha}=0, & \text { and } n=2,3, \ldots, \\ & \text { if } k>n \\ & \text { and } n=1,2, \ldots\end{cases}
$$

Proof. By Lemma 2.1 there exists $M \geq 1$ such that $\left\|E_{\alpha}\left(t^{\alpha} A\right)\right\| \leq M$ and $\left\|E_{\alpha, \alpha}\left(t^{\alpha} A\right)\right\| \leq M$ for all $t \geq 0$. By induction in $n$, we obtain for $\operatorname{Re} \lambda>0$ that $\frac{d^{n}}{d \lambda^{n}}\left(\lambda^{\alpha-1}\left(\lambda^{\alpha}-A\right)^{-1}\right)=(-1)^{n} \lambda^{-(n+1)} \sum_{k=1}^{n+1} b_{k, n+1}^{\alpha}\left[\lambda^{\alpha}\left(\lambda^{\alpha}-A\right)^{-1}\right]^{k}, \quad n=1,2, \ldots$

Furthermore, for each $x \in X$ the function $u(t)=E_{\alpha}\left(t^{\alpha} A\right) x$ is such that

$$
\widehat{u}(\lambda)=\lambda^{\alpha-1}\left(\lambda^{\alpha}-A\right)^{-1} x .
$$

Hence, (2.2) follows by Lemma 2.3.

On the other hand, to prove (2.3), observe that

$$
\frac{d^{n}}{d \lambda^{n}}\left(\lambda^{\alpha}-A\right)^{-1}=(-1)^{n} \lambda^{-n} \sum_{k=1}^{n} \alpha k b_{k, n}^{\alpha} \lambda^{\alpha k}\left(\lambda^{\alpha}-A\right)^{-(k+1)}, \quad n=1,2, \ldots
$$

and for each $x \in X$, the function $v(t)=t^{\alpha-1} E_{\alpha, \alpha}\left(t^{\alpha} A\right) x$ verifies $\widehat{v}(\lambda)=\left(\lambda^{\alpha}-\right.$ $A)^{-1} x$. Using again Lemma 2.3 , the proof is finished.

2.2. Ordered Banach spaces. We recall briefly the basic properties of an order on a Banach space $X$, in order to develop our comparison results.

Definition 2.5. An ordered Banach space is a couple $\left(X, \leq_{X}\right)$ where $X$ is a Banach space and $\leq_{X}$ is an order relation in $X$ such that for every $x, y, z \in X$ and for any scalar $\lambda \geq 0$, it holds that

(a) $x \leq_{X} y$ implies $x+z \leq_{X} y+z$;

(b) $x \leq_{X} y$ implies $\lambda x \leq_{X} \lambda y$; 
(c) the positive cone $C=\left\{x \in X: 0 \leq_{X} x\right\}$ is closed in $X$.

REMARK 2.6. Observe that $x \leq_{X} y$ is equivalent to $0 \leq_{X} y-x$. Furthermore, $x \leq_{X} 0$ if and only if $0 \leq_{X}-x$.

Example 2.7. Consider $1 \leq p \leq \infty$. The Banach spaces $L^{p}(\Omega)$ and $C(\bar{\Omega})$ with the order " $f \leq g$ if and only if $f(x) \leq g(x)$ almost everywhere" are ordered Banach spaces.

Definition 2.8. Suppose that $\left(X, \leq_{X}\right)$ and $\left(Y, \leq_{Y}\right)$ are ordered Banach spaces. A function $T: D(T) \subset X \rightarrow Y$ is called increasing if $x \leq_{X} y$ implies $T x \leq_{Y} T y . T$ is called positive if $0 \leq_{X} x$ implies $0 \leq_{Y} T x$, for all $x, y \in D(T)$.

Lemma 2.9. Let $\left(X, \leq_{X}\right)$ be an ordered Banach space and consider $f \in$ $L^{1}\left(t_{0}, t_{1} ; X\right)$ such that $0 \leq_{X} f(t)$ for almost every $t \in\left(t_{0}, t_{1}\right)$. Then

$$
0 \leq_{X} \int_{t_{0}}^{t_{1}} f(s) d s
$$

\section{Local well posedness}

We start this section with a proof of our result on local existence and uniqueness of mild solutions to (1.1).

Proof of Theorem 1.2. Given $u_{0} \in X$, let $B_{u_{0}}(r)$ be the open ball with center $u_{0}$ and radius $r>0$ and $L=L\left(B_{u_{0}}(r)\right)$ be the Lipschitz constant of $f$ associated to $B_{u_{0}}(r)$. Fix $\beta \in(0, r)$ and choose $t_{0}>0$ such that

$$
(M / \alpha)(L \beta+C) t_{0}^{\alpha} \leq \beta / 2 \quad \text { and } \quad\left\|E_{\alpha}\left(A t^{\alpha}\right) u_{0}-u_{0}\right\| \leq \beta / 2 \quad \text { for all } t \in\left[0, t_{0}\right] .
$$

where $M=\sup _{t \in[0, \infty)}\left\|E_{\alpha, \alpha}\left(A t^{\alpha}\right)\right\|$ and $C=\sup _{t \in\left[0, t_{0}\right]}\left\|f\left(s, u_{0}\right)\right\|$. Consider

$$
K:=\left\{u \in C\left(\left[0, t_{0}\right] ; X\right): u(0)=u_{0} \quad \text { and } \quad\left\|u(t)-u_{0}\right\| \leq \beta \quad \text { for all } t \in\left[0, t_{0}\right]\right\}
$$

and define the operator $T$ on $K$ by

$$
T(u(t))=E_{\alpha}\left(t^{\alpha} A\right) u_{0}+\int_{0}^{t}(t-s)^{\alpha-1} E_{\alpha, \alpha}\left((t-s)^{\alpha} A\right) f(s, u(s)) d s .
$$

If $u \in K$, then $T(u(0))=u_{0}$ and $T(u(t)) \in C\left(\left[0, t_{0}\right] ; X\right)$. Furthermore, we have that

$$
\begin{aligned}
\left\|T(u(t))-u_{0}\right\| \leq & \left\|E_{\alpha}\left(A t^{\alpha}\right) u_{0}-u_{0}\right\| \\
& +\int_{0}^{t}(t-s)^{\alpha-1} M\left(\left\|f(s, u(s))-f\left(s, u_{0}\right)\right\|+\left\|f\left(s, u_{0}\right)\right\|\right) d s \\
\leq & \left\|E_{\alpha}\left(A t^{\alpha}\right) u_{0}-u_{0}\right\|+(M / \alpha)(L \beta+C) t_{0}^{\alpha} \leq \frac{\beta}{2}+\frac{\beta}{2}=\beta,
\end{aligned}
$$

that is, $T(K) \subset K$. Now, if $u, v \in K$, we see that

$$
\|T(u(t))-T(v(t))\| \leq \int_{0}^{t}(t-s)^{\alpha-1} M\|f(s, u(s))-f(s, v(s))\| d s
$$




$$
\begin{aligned}
& \leq \frac{L M t_{0}^{\alpha}}{\alpha} \sup _{s \in\left[0, t_{0}\right]}\|u(s)-v(s)\| \\
& \leq \frac{1}{2} \sup _{s \in\left[0, t_{0}\right]}\|u(s)-v(s)\| .
\end{aligned}
$$

So, by the Banach contraction principle we have that $T$ has a unique fixed point in $K$. We will denote this fixed point by $w$.

Uniqueness of local mild solution to (1.1) follows from the Singular Gronwall Inequality (see [9, Lemma 7.1.1, p. 188]). Therefore, we obtain that the solution $\omega$ is the unique continuous function that satisfies the integral equation and this finishes the proof.

Definition 3.1. Let $u:\left[0, t_{0}\right] \rightarrow X$ be a local mild solution in $\left[0, t_{0}\right]$ to $(1.1)$. If $t_{1}>t_{0}$ and $v:\left[0, t_{1}\right] \rightarrow X$ is a local mild solution to (1.1) in $\left[0, t_{1}\right]$, then we say that $v$ is a continuation of $u$ in $\left[0, t_{1}\right]$.

The remainder of this paragraph will be devoted to the problem of continuation of local mild solutions and existence of global mild solutions of (1.1).

Proof of Theorem 1.3. Let $u:\left[0, t_{0}\right] \rightarrow X$ be the local mild solution to $(1.1)$ in $\left[0, t_{0}\right]$. Since $f$ is locally Lipschitz, there exist $r>0$, an open ball $B=B\left(u\left(t_{0}\right)\right)$ with center in $u\left(t_{0}\right)$ and radius $r$, and $L=L_{u\left(t_{0}\right)}$ the Lipschitz constant of $f$ associated to $B$. Fix $\beta \in(0, r)$ and choose $\tau>0$ such that the following conditions are satisfied:

- $\left\|E_{\alpha}\left(A t^{\alpha}\right) u_{0}-E_{\alpha}\left(A t_{0}{ }^{\alpha}\right) u_{0}\right\| \leq \beta / 4$,

- $(M / \alpha)(L \beta+C) \tau^{\alpha} \leq \beta / 4, \quad(M D / \alpha)\left[t^{\alpha}-\left(t-t_{0}\right)^{\alpha}-t_{0}^{\alpha}\right] \leq \beta / 4$,

- $\int_{0}^{t_{0}}\left(t_{0}-s\right)^{\alpha-1}\left\|\left[E_{\alpha, \alpha}\left(A(t-s)^{\alpha}\right)-E_{\alpha, \alpha}\left(A\left(t_{0}-s\right)^{\alpha}\right)\right] f(s, u(s))\right\| d s \leq \frac{\beta}{4}$

for all $t \in\left[t_{0}, t_{0}+\tau\right]$, where

$$
\begin{aligned}
C & =\sup _{s \in\left[0, t_{0}\right]}\left\|f\left(s, u\left(t_{0}\right)\right)\right\|, \\
D & =\sup _{s \in\left[0, t_{0}\right]}\|f(s, u(s))\|, \\
M & =\max \left\{\sup _{t \in[0, \infty)}\left\|E_{\alpha, \alpha}\left(A t^{\alpha}\right)\right\|, \sup _{t \in[0, \infty)}\left\|E_{\alpha}\left(A t^{\alpha}\right)\right\|\right\} .
\end{aligned}
$$

Consider

$$
\begin{aligned}
K:=\left\{w \in C\left(\left[0, t_{0}+\tau\right] ; X\right):\right. & w(t)=u(t) \text { for all } t \in\left[0, t_{0}\right] \\
& \text { and } \left.\left\|w(t)-u\left(t_{0}\right)\right\| \leq \beta \text { for all } t \in\left[t_{0}, t_{0}+\tau\right]\right\}
\end{aligned}
$$

and $T: K \rightarrow C\left(\left[0, t_{0}+\tau\right] ; X\right)$ given by

$$
T(w(t))=E_{\alpha}\left(t^{\alpha} A\right) u_{0}+\int_{0}^{t}(t-s)^{\alpha-1} E_{\alpha, \alpha}\left((t-s)^{\alpha} A\right) f(s, u(s)) d s .
$$


454 B. de Andrade - A.N. Carvalho - P.M. Carvalho-Neto - P. Marín-Rubio

We check that $T(K) \subset K$.

(a) If $w \in K$, then $w(t)=u(t)$ in $\left[0, t_{0}\right]$ with $\mathrm{u}$ the local mild solution to (1.1) in $\left[0, t_{0}\right]$. So, if $t \in\left[0, t_{0}\right]$,

$$
\begin{aligned}
T(w(t)) & =E_{\alpha}\left(t^{\alpha} A\right) u_{0}+\int_{0}^{t}(t-s)^{\alpha-1} E_{\alpha, \alpha}\left((t-s)^{\alpha} A\right) f(s, w(s)) d s \\
& =E_{\alpha}\left(t^{\alpha} A\right) u_{0}+\int_{0}^{t}(t-s)^{\alpha-1} E_{\alpha, \alpha}\left((t-s)^{\alpha} A\right) f(s, u(s)) d s=u(t) .
\end{aligned}
$$

(b) If $t \in\left[t_{0}, t_{0}+\tau\right]$, then

$$
\begin{aligned}
\| T(w(t)) & -u\left(t_{0}\right)\|\leq\| E_{\alpha}\left(t^{\alpha} A\right) u_{0}-E_{\alpha}\left(t_{0}{ }^{\alpha} A\right) u_{0} \| \\
& +\| \int_{0}^{t}(t-s)^{\alpha-1} E_{\alpha, \alpha}\left((t-s)^{\alpha} A\right) f(s, w(s)) d s \\
& \quad-\int_{0}^{t_{0}}\left(t_{0}-s\right)^{\alpha-1} E_{\alpha, \alpha}\left(\left(t_{0}-s\right)^{\alpha} A\right) f(s, w(s)) d s \| \\
\leq & \mathcal{I}_{1}+\mathcal{I}_{2}+\mathcal{I}_{3}+\mathcal{I}_{4} \leq \beta,
\end{aligned}
$$

where

$$
\begin{aligned}
& \mathcal{I}_{1}=\left\|E_{\alpha}\left(t^{\alpha} A\right) u_{0}-E_{\alpha}\left(t_{0}{ }^{\alpha} A\right) u_{0}\right\| \leq \frac{\beta}{4}, \\
& \mathcal{I}_{2}=\left\|\int_{t_{0}}^{t}(t-s)^{\alpha-1} E_{\alpha, \alpha}\left((t-s)^{\alpha} A\right) f(s, w(s)) d s\right\| \leq \frac{\beta}{4}, \\
& \mathcal{I}_{3}=\left\|\int_{0}^{t_{0}}\left[(t-s)^{\alpha-1}-\left(t_{0}-s\right)^{\alpha-1}\right] E_{\alpha, \alpha}\left((t-s)^{\alpha} A\right) f(s, w(s)) d s\right\| \leq \frac{\beta}{4}, \\
& \mathcal{I}_{4}=\left\|\int_{0}^{t_{0}}\left(t_{0}-s\right)^{\alpha-1}\left[E_{\alpha, \alpha}\left((t-s)^{\alpha} A\right)-E_{\alpha, \alpha}\left(\left(t_{0}-s\right)^{\alpha} A\right)\right] f(s, w(s)) d s\right\| \leq \frac{\beta}{4} .
\end{aligned}
$$

By similar computations, we conclude that, for every $t \in\left[0, t_{0}+\tau\right]$,

$$
\begin{aligned}
\|T(\omega(t))-T(v(t))\| & \leq \frac{L M \tau^{\alpha}}{\alpha} \sup _{s \in\left[0, t_{0}+\tau\right]}\|\omega(s)-v(s)\| \\
& \leq \frac{1}{2} \sup _{s \in\left[0, t_{0}+\tau\right]}\|\omega(s)-v(s)\|
\end{aligned}
$$

for all $u, v \in K$. Therefore, by the Banach contraction principle, we conclude that there exists a unique fixed point $u^{*} \in K$ of the integral equation. As in the proof of Theorem 1.2, it is not difficult to see that $u^{*}$ is the unique continuous continuation of $u$ in $\left[0, t_{0}+\tau\right]$.

The following result will be useful in the proof of Theorem 1.4.

Lemma 3.2. Consider $\omega \in(0, \infty), u:[0, \omega) \rightarrow X$ a bounded continuous function, and $f:[0, \infty) \times X \rightarrow X$ continuous and bounded. If $\left\{t_{n}\right\} \subset[0, \omega)$ 
satisfies $\lim _{n} t_{n}=\omega$, then

$$
\lim _{n} \int_{0}^{t_{n}}\left(t_{n}-r\right)^{\alpha-1}\left\|\left[E_{\alpha, \alpha}\left(A\left(t_{n}-r^{\alpha}\right)\right)-E_{\alpha, \alpha}\left(A\left(w-r^{\alpha}\right)\right)\right] f(r, u(r))\right\| d r=0 .
$$

Proof. Let $M=\sup _{t \in[0, \infty)}\left\|E_{\alpha, \alpha}\left(A t^{\alpha}\right)\right\|$ and $K=\sup _{s \in[0, \omega)}\|f(s, u(s))\|$. Given $\varepsilon>0$, fix $\gamma \in(0, \omega)$ such that

$$
\frac{(\omega-\gamma)^{\alpha}}{\alpha} M K \leq \frac{\varepsilon}{2}
$$

Now, choose $N \in \mathbb{N}$ such that

- $t_{n}>\gamma$ for all $n \geq N$;

- $\int_{0}^{\gamma}\left(t_{n}-r\right)^{\alpha-1}\left\|\left[E_{\alpha, \alpha}\left(A\left(t_{n}-r^{\alpha}\right)\right)-E_{\alpha, \alpha}\left(A\left(w-r^{\alpha}\right)\right)\right] f(r, u(r))\right\| d r<\frac{\varepsilon}{2}$ for all $n \geq N$.

Hence, we conclude that for $n \geq N$,

$$
\begin{aligned}
\int_{0}^{t_{n}}\left(t_{n}-r\right)^{\alpha-1} & \left\|\left[E_{\alpha, \alpha}\left(A\left(t_{n}-r^{\alpha}\right)\right)-E_{\alpha, \alpha}\left(A\left(w-r^{\alpha}\right)\right)\right] f(r, u(r))\right\| d r \\
\leq & \frac{\varepsilon}{2}+\int_{\gamma}^{t_{n}}\left(t_{n}-r\right)^{\alpha-1} M K d r \leq \frac{\varepsilon}{2}+\frac{(\omega-\gamma)^{\alpha}}{\alpha} M K \leq \varepsilon
\end{aligned}
$$

Proof of Theorem 1.4. Consider $H:=\left\{\tau \in[0, \infty)\right.$ : there exists $u_{\tau}:[0, \tau]$ $\rightarrow X$ unique local mild solution to (1.1) in $[0, \tau]\}$. If $\sup H=w$, we can consider a continuous function $u:[0, \omega) \rightarrow X$ that is a local mild solution to (1.1) in $[0, \omega)$. If $\omega=\infty$, then $u$ is a global mild solution in $[0, \infty)$. Otherwise, if $\omega<\infty$ we will prove that $\lim \sup |u(t)|=\infty$. By contradiction, suppose that there exists $K<\infty$ such that $\|u(t)\| \leq K$ for all $t \in[0, \omega)$. Therefore, it follows from Lemma 3.2 that if $\left\{t_{n}\right\} \subset[0, \omega)$ is a sequence that converges to $\omega$, given $\varepsilon>0$, there exists $N \in \mathbb{N}$, such that, if $m, n \geq N$, we have

$$
\begin{gathered}
\left\|E_{\alpha}\left(t_{n}^{\alpha} A\right) u_{0}-E_{\alpha}\left(t_{m}^{\alpha} A\right) u_{0}\right\| \leq \frac{\varepsilon}{5} \\
\left|t_{n}-t_{m}\right|^{\alpha} \frac{M K}{\alpha} \leq \frac{\varepsilon}{5} \\
\left|t_{n}^{\alpha}-\left(t_{n}-t_{m}\right)^{\alpha}-t_{m}^{\alpha}\right| \frac{M K}{\alpha} \leq \frac{\varepsilon}{5} \\
\int_{0}^{t_{n}}\left(t_{n}-r\right)^{\alpha-1}\left\|\left[E_{\alpha, \alpha}\left(\left(t_{n}-r\right)^{\alpha} A\right)-E_{\alpha, \alpha}\left((w-r)^{\alpha} A\right)\right] f(r, u(r))\right\| d r \leq \frac{\varepsilon}{5}, \\
\int_{0}^{t_{m}}\left(t_{m}-r\right)^{\alpha-1}\left\|\left[E_{\alpha, \alpha}\left(\left(t_{m}-r\right)^{\alpha} A\right)-E_{\alpha, \alpha}\left((w-r)^{\alpha} A\right)\right] f(r, u(r))\right\| d r \leq \frac{\varepsilon}{5},
\end{gathered}
$$

where

$$
K=\sup _{t \in[0, \omega)}\|f(t, u(t))\|, \quad M=\max \left\{\sup _{t \in[0, \infty)}\left\|E_{\alpha, \alpha}\left(A t^{\alpha}\right)\right\|, \quad \sup _{t \in[0, \infty)}\left\|E_{\alpha}\left(A t^{\alpha}\right)\right\|\right\} .
$$


456 B. de Andrade - A.N. Carvalho - P.M. Carvalho-Neto - P. Marín-Rubio

Hence, for $n, m \geq N$ and assuming, without loss of generality, that $t_{n}>t_{m}$, it follows from the estimate

$$
\left\|u\left(t_{n}\right)-u\left(t_{m}\right)\right\| \leq\left\|E_{\alpha}\left(t_{n}^{\alpha} A\right) u_{0}-E_{\alpha}\left(t_{m}^{\alpha} A\right) u_{0}\right\|+\mathcal{I}_{1}+\mathcal{I}_{2}+\mathcal{I}_{3},
$$

where

$$
\begin{aligned}
\mathcal{I}_{1}= & \int_{t_{m}}^{t_{n}}\left(t_{n}-r\right)^{\alpha-1}\left\|E_{\alpha, \alpha}\left(\left(t_{n}-r\right)^{\alpha} A\right) f(r, u(r))\right\| d r \leq\left|t_{n}-t_{m}\right|^{\alpha} \frac{M K}{\alpha}, \\
\mathcal{I}_{2}= & \int_{0}^{t_{m}}\left(t_{n}-r\right)^{\alpha-1}\left\|\left[E_{\alpha, \alpha}\left(\left(t_{n}-r\right)^{\alpha} A\right)-E_{\alpha, \alpha}\left(\left(t_{m}-r\right)^{\alpha} A\right)\right] f(r, u(r))\right\| d r \\
\leq & \int_{0}^{t_{m}}\left(t_{n}-r\right)^{\alpha-1}\left\|\left[E_{\alpha, \alpha}\left(\left(t_{n}-r\right)^{\alpha} A\right)-E_{\alpha, \alpha}\left((\omega-r)^{\alpha} A\right)\right] f(r, u(r))\right\| d r \\
& +\int_{0}^{t_{m}}\left(t_{n}-r\right)^{\alpha-1}\left\|\left[E_{\alpha, \alpha}\left(\left(t_{m}-r\right)^{\alpha} A\right)-E_{\alpha, \alpha}\left((\omega-r)^{\alpha} A\right)\right] f(r, u(r))\right\| d r \\
\leq & \int_{0}^{t_{n}}\left(t_{n}-r\right)^{\alpha-1}\left\|\left[E_{\alpha, \alpha}\left(\left(t_{n}-r\right)^{\alpha} A\right)-E_{\alpha, \alpha}\left((\omega-r)^{\alpha} A\right)\right] f(r, u(r))\right\| d r \\
& +\int_{0}^{t_{m}}\left(t_{m}-r\right)^{\alpha-1}\left\|\left[E_{\alpha, \alpha}\left(\left(t_{m}-r\right)^{\alpha} A\right)-E_{\alpha, \alpha}\left((\omega-r)^{\alpha} A\right)\right] f(r, u(r))\right\| d r
\end{aligned}
$$

and

$$
\begin{aligned}
\mathcal{I}_{3} & =\int_{0}^{t_{m}}\left|\left[\left(t_{n}-s\right)^{\alpha-1}-\left(t_{m}-s\right)^{\alpha-1}\right]\right|\left\|E_{\alpha, \alpha}\left(\left(t_{m}-s\right)^{\alpha} A\right) f(s, u(s))\right\| d s \\
& \leq\left|t_{n}^{\alpha}-\left(t_{n}-t_{m}\right)^{\alpha}-t_{m}^{\alpha}\right| \frac{M K}{\alpha},
\end{aligned}
$$

that $\left\|u\left(t_{n}\right)-u\left(t_{m}\right)\right\| \leq \varepsilon$. This computation shows that $\left\{u\left(t_{n}\right)\right\}$ is a Cauchy sequence and therefore it has a limit, $u_{t} \in X$. Then, we may extend $u$ over $[0, \omega]$ obtaining the equality

$$
u(t)=E_{\alpha}\left(t^{\alpha} A\right) u_{0}+\int_{0}^{t}(t-s)^{\alpha-1} E_{\alpha, \alpha}\left((t-s)^{\alpha} A\right) f(s, u(s)) d s
$$

for all $t \in[0, \omega]$. With this, by Theorem 1.3 , we can extend the solution to some bigger interval, which is a contradiction with the definition of $\omega$.

Corollary 3.3. Let $f:[0, \infty) \times X \rightarrow X$ be as in Theorem 1.4 and suppose that there exists a constant $K>0$ such that if $\left\|u_{0}\right\| \leq K$, then the solutions to (1.1), while exists, are bounded by $K$. Then (1.1) possesses a unique global mild solution.

\section{Critical nonlinearities}

Following the notation of [2], we consider the following class of nonlinearities: let $\varepsilon, \gamma(\varepsilon), \xi, \zeta, c$, and $\delta$ be positive constants, and a function $\nu$ with values in $[0, \delta)$ and $\lim _{t \rightarrow 0^{+}} \nu(t)=0$. Define $\mathcal{F}(\varepsilon, \rho, \gamma(\varepsilon), c, \nu(\cdot), \xi, \zeta)$ as the family of 
functions $f$ such that, for $t \geq 0, f(t, \cdot)$ is an $\varepsilon$-regular map relative to the pair $\left(X^{1}, X^{0}\right)$, satisfying, for all $x, y \in X^{1+\varepsilon}$,

$$
\begin{aligned}
\|f(t, x)-f(t, y)\|_{X^{\gamma(\varepsilon)}} & \leq c\left(\|x\|_{X^{1+\varepsilon}}^{\rho-1}+\|y\|_{X^{1+\varepsilon}}^{\rho-1}+\nu(t) t^{-\zeta}\right)\|x-y\|_{X^{1+\varepsilon}} \\
\|f(t, x)\|_{X^{\gamma(\varepsilon)}} & \leq c\left(\|x\|_{X^{1+\varepsilon}}^{\rho}+\nu(t) t^{-\xi}\right) .
\end{aligned}
$$

Without loss of generality we may assume that the function $\nu$ is non-decreasing. We will suppose that $0 \leq \zeta \leq \alpha(\gamma(\varepsilon)-\varepsilon)$ and $0 \leq \xi \leq \alpha \gamma(\varepsilon), \alpha \in(0,1)$. In most cases in the arguments below we will fix the parameters $\varepsilon, \gamma(\varepsilon), \rho, \xi, \zeta$ and $c$, and we will denote the class $\mathcal{F}$ defined above by $\mathcal{F}(\nu(\cdot))$.

As immediate consequence of Lemma 2.2 we have that if $0 \leq \theta, \beta \leq 1$, then

$$
\begin{aligned}
t^{\alpha(1+\theta-\beta)}\left\|E_{\alpha}\left(t^{\alpha} A\right) x\right\|_{X^{1+\theta}} & \leq M\|x\|_{X^{\beta}}, \\
t^{\alpha(\theta-\beta)+1}\left\|t^{\alpha-1} E_{\alpha, \alpha}\left(t^{\alpha} A\right) x\right\|_{X^{1+\theta}} & \leq M\|x\|_{X^{\beta}} .
\end{aligned}
$$

To prove Theorem 1.7 we need some previous results.

Lemma 4.1. Consider $\alpha \in(0,1), \theta \in[0,1]$, and a sectorial operator $A$. The operators $\left\{t^{\alpha \theta} E_{\alpha}\left(t^{\alpha} A\right): X^{1} \rightarrow X^{1+\theta}\right\}_{t>0}$ are bounded linear operators satisfying

$$
\left\|t^{\alpha \theta} E_{\alpha}\left(t^{\alpha} A\right)\right\|_{\mathcal{L}\left(X^{1}, X^{1+\theta}\right)} \leq M,
$$

with $M>0$ independent of $t$. Moreover, given a compact subset $J$ of $X^{1}$, we have

$$
\lim _{t \rightarrow 0^{+}} \sup _{x \in J}\left\|t^{\alpha \theta} E_{\alpha}\left(t^{\alpha} A\right) x\right\|_{X^{1+\theta}}=0 .
$$

Proof. The fact that $\left\|t^{\alpha \theta} E_{\alpha}\left(t^{\alpha} A\right)\right\|_{\mathcal{L}\left(X^{1}, X^{1+\theta}\right)} \leq M$ follows from (4.1). For the remaining part it suffices to observe that the operators $t^{\alpha} E_{\alpha}\left(t^{\alpha} A\right): X^{1} \rightarrow$ $X^{1+\alpha}$ are uniformly bounded in $t$, that

$$
\lim _{t \rightarrow 0^{+}}\left\|t^{\alpha} E_{\alpha}\left(t^{\alpha} A\right) x\right\|_{X^{1+\alpha}}=0,
$$

for $x \in X^{1+\alpha}$, and that $X^{\alpha}$ is a dense subset of $X^{1}$.

Let $\mathbf{B}:(0, \infty) \times(0, \infty) \rightarrow(0, \infty)$ be the beta function given by

$$
\mathbf{B}(a, b)=\int_{0}^{1}(1-x)^{a-1} x^{b-1} d x .
$$

For $\zeta \geq 0$, define

$$
\mathbf{B}_{\varepsilon}^{\theta}(\zeta)=\sup _{0 \leq \eta \leq \theta}\{\mathbf{B}(\alpha(\gamma(\varepsilon)-\eta), 1-\zeta), \mathbf{B}(\alpha(\gamma(\varepsilon)-\eta), 1-\alpha \rho \varepsilon)\} .
$$

Lemma 4.2. With the above notation, let $f \in \mathcal{F}(\nu(\cdot))$. If $u \in C\left((0, \tau] ; X^{1+\varepsilon}\right)$, then, for all $0 \leq \theta<\gamma(\varepsilon)$, we have that

$$
\begin{array}{r}
t^{\alpha \theta}\left\|\int_{0}^{t}(t-s)^{\alpha-1} E_{\alpha, \alpha}\left((t-s)^{\alpha} A\right) f(s, u(s)) d s\right\|_{X^{1+\theta}} \\
\leq M c \mathbf{B}_{\varepsilon}^{\theta}(\xi)\left(\nu(t) t^{\alpha \gamma(\varepsilon)-\xi}+\lambda(t)^{\rho} t^{\alpha(\gamma(\varepsilon)-\rho \varepsilon)}\right)
\end{array}
$$


458 B. de Andrade - A.N. Carvalho - P.M. Carvalho-Neto - P. Marín-Rubio for $0<t \leq \tau$, where $\lambda(t)=\sup _{s \in(0, t]}\left\{s^{\alpha \varepsilon}\|u(s)\|_{X^{1+\varepsilon}}\right\}$.

Proof. Indeed, we have that

$$
\begin{aligned}
& t^{\alpha \theta} \int_{0}^{t}\left\|(t-s)^{\alpha-1} E_{\alpha, \alpha}\left((t-s)^{\alpha} A\right) f(s, u(s))\right\|_{X^{1+\theta}} d s \\
& \leq M t^{\alpha \theta} \int_{0}^{t}(t-s)^{\alpha(\gamma(\varepsilon)-\theta)-1} c\left(\nu(s) s^{-\xi}+\|u(s)\|_{X^{1+\varepsilon}}^{\rho}\right) d s \\
& \leq M c \nu(t) t^{\alpha \theta} \int_{0}^{t}(t-s)^{\alpha(\gamma(\varepsilon)-\theta)-1} s^{-\xi} d s \\
& \quad+M c t^{\alpha \theta} \int_{0}^{t}(t-s)^{\alpha(\gamma(\varepsilon)-\theta)-1} s^{-\alpha \rho \varepsilon}\left(s^{\alpha \varepsilon}\|u(s)\|_{X^{1+\varepsilon}}\right)^{\rho} d s \\
& \leq M c \nu(t) t^{\alpha \theta-\xi+\alpha(\gamma(\varepsilon)-\theta)} \int_{0}^{1}(1-s)^{\alpha(\gamma(\varepsilon)-\theta)-1} s^{-\xi} d s \\
& \quad+M c \lambda(t)^{\rho} t^{\alpha \theta-\alpha \rho \varepsilon+\alpha(\gamma(\varepsilon)-\theta)} \int_{0}^{1}(t-s)^{\alpha(\gamma(\varepsilon)-\theta)-1} s^{-\alpha \rho \varepsilon} d s \\
& \leq M c \mathbf{B}_{\varepsilon}^{\theta}(\xi)\left(\nu(t) t^{\alpha \gamma(\varepsilon)-\xi}+\lambda(t)^{\rho} t^{\alpha(\gamma(\varepsilon)-\rho \varepsilon)}\right),
\end{aligned}
$$

which concludes the proof.

Lemma 4.3. With the above notation, let $f \in \mathcal{F}(\nu(\cdot))$ and consider $u, v \in$ $C\left((0, \tau] ; X^{1+\varepsilon}\right)$ such that $t^{\alpha \varepsilon}\|u(t)\|_{X^{1+\varepsilon}} \leq \mu$ and $t^{\alpha \varepsilon}\|v(t)\|_{X^{1+\varepsilon}} \leq \mu$ for some $\mu>0$. Then, for all $0 \leq \theta<\gamma(\varepsilon)<1$, we have

$$
\begin{aligned}
t^{\alpha \theta} \| \int_{0}^{t}(t-s)^{\alpha-1} E_{\alpha, \alpha}\left((t-s)^{\alpha} A\right)(f(s, u(s)) & -f(s, v(s))) d s \|_{X^{1+\theta}} \\
\leq M c \mathbf{B}_{\varepsilon}^{\theta}(\zeta+\alpha \varepsilon)\left[\nu(t) t^{\alpha(\gamma(\varepsilon)-\theta)-\zeta}+\right. & \left.2 \mu^{\rho-1} t^{\alpha(\gamma(\varepsilon)+\varepsilon-\theta-\rho \varepsilon)}\right] \\
& \times \sup _{0<s \leq \tau} s^{\alpha \varepsilon}\|u(s)-v(s)\|_{X^{1+\varepsilon}} .
\end{aligned}
$$

Proof. It follows from the $\varepsilon$-regularity property of $f$ that

$$
\begin{aligned}
& t^{\alpha \theta} \int_{0}^{t}\left\|(t-s)^{\alpha-1} E_{\alpha, \alpha}\left((t-s)^{\alpha} A\right)(f(s, u(s))-f(s, v(s)))\right\|_{X^{1+\theta}} d s \\
& \leq M t^{\alpha \theta} \int_{0}^{t}(t-s)^{\alpha(\gamma(\varepsilon)-\theta)-1}\|f(s, u(s))-f(s, v(s))\|_{X^{\gamma(\varepsilon)}} d s \\
& \leq M c t^{\alpha \theta} \int_{0}^{t}(t-s)^{\alpha(\gamma(\varepsilon)-\theta)-1}\|u(s)-v(s)\|_{X^{1+\varepsilon}} \\
& \quad \times\left(\|u(s)\|_{X^{1+\varepsilon}}^{\rho-1}+\|v(s)\|_{X^{1+\varepsilon}}^{\rho-1}+\nu(s) s^{-\zeta}\right) d s \\
& \leq M c \nu(t) t^{\alpha \theta} \int_{0}^{t}(t-s)^{\alpha(\gamma(\varepsilon)-\theta)-1} s^{-\zeta-\alpha \varepsilon} s^{\alpha \varepsilon}\|u(s)-v(s)\|_{X^{1+\varepsilon}} d s \\
&+M c t^{\alpha \theta} \int_{0}^{t}(t-s)^{\alpha(\gamma(\varepsilon)-\theta)-1} s^{-\alpha \rho \varepsilon}
\end{aligned}
$$




$$
\begin{aligned}
& \times\left[\left(s^{\alpha \varepsilon}\|u(s)\|_{X^{1+\varepsilon}}\right)^{\rho-1}+\left(s^{\alpha \varepsilon}\|v(s)\|_{X^{1+\varepsilon}}\right)^{\rho-1}\right] s^{\alpha \varepsilon}\|u(s)-v(s)\|_{x^{1+\varepsilon}} d s \\
\leq & {\left[\nu(t) t^{\alpha(\gamma(\varepsilon)-\theta)-\zeta}+2 \mu^{\rho-1} t^{\alpha(\gamma(\varepsilon)+\varepsilon-\theta-\rho \varepsilon)}\right] } \\
& \times M c \mathbf{B}_{\varepsilon}^{\theta}(\zeta+\alpha \varepsilon) \sup _{0 \leq s \leq \tau} s^{\alpha \varepsilon}\|u(s)-v(s)\|_{X^{1+\varepsilon}}
\end{aligned}
$$

Now we may prove the main result of this section.

Proof of Theorem 1.7. Define $\mu$ by

$$
M c \mathbf{B}_{\varepsilon}^{\varepsilon} \mu^{\rho-1}=\frac{1}{8},
$$

where $\mathbf{B}_{\varepsilon}^{\theta}:=\max \left\{\mathbf{B}_{\varepsilon}^{\theta}(\xi), \mathbf{B}_{\varepsilon}^{\theta}(\zeta+\alpha \varepsilon)\right\}$ and choose $r=r(\mu, M)>0$ such that

$$
r=\frac{\mu}{4 M}=\frac{1}{4 M\left(8 c M \mathbf{B}_{\varepsilon}^{\varepsilon}\right)^{1 /(\rho-1)}} .
$$

For $v_{0}$ fixed, choose $\tau_{0} \in(0,1]$ such that $\nu(t)<\delta$ for all $t \in\left(0, \tau_{0}\right]$,

$$
\left\|t^{\alpha \varepsilon} E_{\alpha}\left(t^{\alpha} A\right) v_{0}\right\|_{X^{1+\varepsilon}} \leq \frac{\mu}{2} \quad \text { if } 0 \leq t \leq \tau_{0},
$$

and

Consider

$$
M c \delta \mathbf{B}_{\varepsilon}^{\varepsilon}=\min \left\{\frac{\mu}{8}, \frac{1}{4}\right\}
$$

$$
K\left(\tau_{0}\right)=\left\{u \in C\left(\left(0, \tau_{0}\right] ; X^{1+\varepsilon}\right): \sup _{t \in\left(0, \tau_{0}\right]} t^{\alpha \varepsilon}\|u(t)\|_{X^{1+\varepsilon}} \leq \mu\right\}
$$

with norm

$$
\|u\|_{K\left(\tau_{0}\right)}=\sup _{t \in\left(0, \tau_{0}\right]} t^{\alpha \varepsilon}\|u(t)\|_{X^{1+\epsilon}} .
$$

Suppose that $u_{0} \in X^{1}$ with $\left\|u_{0}-v_{0}\right\|_{X^{1}}<r$ and define on $K\left(\tau_{0}\right)$ the map

$$
T u(t)=E_{\alpha}\left(t^{\alpha} A\right) u_{0}+\int_{0}^{t}(t-s)^{\alpha-1} E_{\alpha, \alpha}\left((t-s)^{\alpha} A\right) f(s, u(s)) d s .
$$

Our purpose is to show that for any $u_{0} \in B_{X^{1}}\left(v_{0}, r\right), K\left(\tau_{0}\right)$ is $T$-invariant and $T$ is a contraction.

Initially, let us prove that $T: K\left(\tau_{0}\right) \rightarrow K\left(\tau_{0}\right)$ is well defined.

Claim 1. If $u \in K\left(\tau_{0}\right)$, then $T u \in C\left(\left(0, \tau_{0}\right] ; X^{1+\theta}\right)$ for all $\theta \in[0, \gamma(\varepsilon))$.

For this, let $t_{1}, t_{2} \in\left(0, \tau_{0}\right], t_{1}>t_{2}$; then, for every $0 \leq \theta<\gamma(\varepsilon)$, we have that

$$
\begin{aligned}
\| T u\left(t_{1}\right), & -T u\left(t_{2}\right)\left\|_{X^{1+\theta}} \leq\right\|\left(E_{\alpha}\left(t_{1}^{\alpha} A\right)-E_{\alpha}\left(t_{2}^{\alpha} A\right)\right) u_{0} \|_{X^{1+\theta}} \\
& +\| \int_{0}^{t_{2}}\left(\left(t_{1}-s\right)^{\alpha-1} E_{\alpha, \alpha}\right. \\
& \left.\times\left(\left(t_{2}-s\right)^{\alpha} A\right)-\left(t_{1}-s\right)^{\alpha-2} E_{\alpha, \alpha}\left(\left(t_{2}-s\right)^{\alpha} A\right)\right) f(s, u(s)) d s \|_{X^{1+\theta}} \\
& +\left\|\int_{t_{2}}^{t_{1}}\left(t_{1}-s\right)^{\alpha-1} E_{\alpha, \alpha}\left(\left(t_{1}-s\right)^{\alpha} A\right) f(s, u(s)) d s\right\|_{X^{1+\theta}} \cdot
\end{aligned}
$$


460 B. de Andrade - A.N. Carvalho - P.M. Carvalho-Neto - P. Marín-Rubio

The first term trivially goes to 0 as $t_{1} \rightarrow t_{2}^{+}$. A similar procedure to Theorem 1.4 shows that the second term also goes to 0 as $t_{1} \rightarrow t_{2}^{+}$. Let us consider the third term. We have

$$
\begin{aligned}
\left\|\int_{t_{2}}^{t_{1}}\left(t_{1}-s\right)^{\alpha-1} E_{\alpha, \alpha}\left(\left(t_{1}-s\right)^{\alpha} A\right) f(s, u(s)) d s d s\right\|_{X^{1+\theta}} \\
\leq M \int_{t_{2}}^{t_{1}}\left(t_{1}-s\right)^{\alpha(\gamma(\varepsilon)-\theta)-1}\|f(s, u(s))\|_{X^{\gamma(\varepsilon)}} d s \\
\leq M c \int_{t_{2}}^{t_{1}}\left(t_{1}-s\right)^{\alpha(\gamma(\varepsilon)-\theta)-1}\left(\nu(s) s^{-\xi}+\|u(s)\|_{X^{1+\varepsilon}}^{\rho-1}\right) d s \\
\leq M c \delta \int_{t_{2}}^{t_{1}}\left(t_{1}-s\right)^{\alpha(\gamma(\varepsilon)-\theta)-1} s^{-\xi} d s \\
\quad+M c \int_{t_{2}}^{t_{1}}\left(t_{1}-s\right)^{\alpha(\gamma(\varepsilon)-\theta)-1}\|u(s)\|_{X^{1+\varepsilon}} d s \\
\leq M c \delta t_{1}^{\alpha(\gamma(\varepsilon)-\theta)-\xi} \int_{t_{2} / t_{1}}^{1}(1-s)^{\alpha(\gamma(\varepsilon)-\theta)-1} s^{-\xi} d s \\
\quad+M c \mu^{\rho} t^{\alpha(\gamma(\varepsilon)-\theta-\rho \varepsilon)} \int_{t_{2} / t_{1}}^{1}(1-s)^{\alpha(\gamma(\varepsilon)-\theta)-1} s^{-\alpha \rho \varepsilon} d s
\end{aligned}
$$

which goes to 0 as $t_{1} \rightarrow t_{2}^{+}$. The case $t_{1}<t_{2}$ is analogous.

Claim 2. $t^{\alpha \varepsilon}\|T u(t)\|_{X^{1+\varepsilon}} \leq \mu$ for all $t \in\left(0, \tau_{0}\right]$.

Indeed, we may estimate as follows.

$$
\begin{aligned}
t^{\alpha \varepsilon} & \|T u(t)\|_{X^{1+\varepsilon}} \leq\left\|t^{\alpha \varepsilon} E_{\alpha}\left(A t^{\alpha}\right) u_{0}\right\|_{X^{1+\varepsilon}} \\
& +M t^{\alpha \varepsilon} \int_{0}^{t}(t-s)^{\alpha(\gamma(\varepsilon)-\varepsilon)-1}\|f(s, u(s))\|_{X^{\gamma(\varepsilon)}} d s \\
\leq & \left\|t^{\alpha \varepsilon} E_{\alpha}\left(A t^{\alpha}\right)\left(u_{0} \mp v_{0}\right)\right\|_{X^{1+\varepsilon}} \\
& +M c t^{\alpha \varepsilon} \int_{0}^{t}(t-s)^{\alpha(\gamma(\varepsilon)-\epsilon)-1}\left(\nu(s) s^{-\xi}+\|u(s)\|_{X^{1+\varepsilon}}^{\rho}\right) d s \\
\leq & M r+\left\|t^{\alpha \varepsilon} E_{\alpha}\left(A t^{\alpha}\right) v_{0}\right\|_{X^{1+\varepsilon}}+M c \delta t^{\alpha \gamma(\varepsilon)-\xi} \int_{0}^{1}(1-s)^{\alpha(\gamma(\varepsilon)-\varepsilon)-1} s^{-\xi} d s \\
& +M c \mu^{\rho} t^{\alpha(\gamma(\varepsilon)-\rho \varepsilon)} \int_{0}^{1}(1-s)^{\alpha(\gamma(\varepsilon)-\varepsilon)-1} s^{-\alpha \rho \varepsilon} d s \\
\leq & M r+\left\|t^{\alpha \varepsilon} E_{\alpha}\left(A t^{\alpha}\right) v_{0}\right\|_{X^{1+\varepsilon}}+M c \delta \mathbf{B}_{\varepsilon}^{\varepsilon}+M c \mu^{\rho} \mathbf{B}_{\varepsilon}^{\varepsilon} \leq \mu .
\end{aligned}
$$

This shows that $T\left(K\left(\tau_{0}\right)\right) \subset K\left(\tau_{0}\right)$.

Finally, by taking $\theta=\varepsilon$, it follows from Lemma 4.3 that $T$ is a strict contraction in $K\left(\tau_{0}\right)$ and that

$$
\|T u(t)-T v(t)\|_{K\left(\tau_{0}\right)} \leq \frac{1}{2}\|u-v\|_{K\left(\tau_{0}\right)} .
$$


By the Banach contraction principle we have that $T$ has a unique fixed point in $K\left(\tau_{0}\right)$, which will be denoted by $u\left(\cdot, u_{0}\right)$. It is defined for $\left\|u_{0}-v_{0}\right\|_{X^{1}}<r$, $0 \leq t \leq \tau_{0}$.

Observe that $u\left(\cdot, u_{0}\right) \in C\left(\left(0, \tau_{0}\right] ; X^{1+\theta}\right)$ for all $0 \leq \theta<\gamma(\varepsilon)$. Furthermore, we will prove that

Claim 3. $t^{\alpha \theta}\left\|u\left(t, u_{0}\right)\right\|_{X^{1+\theta}} \rightarrow 0$ as $t \rightarrow 0$, for all $0<\theta<\gamma(\varepsilon)$.

Indeed, from Lemma 4.2 we have that

$$
\begin{aligned}
t^{\alpha \theta}\left\|u\left(t, u_{0}\right)\right\|_{X^{1+\theta}} \leq & t^{\alpha \theta}\left\|E_{\alpha}\left(t^{\alpha} A\right) u_{0}\right\|_{X^{1+\theta}} \\
& +t^{\alpha \theta}\left\|\int_{0}^{t}(t-s)^{\alpha-1} E_{\alpha, \alpha}\left((t-s)^{\alpha} A\right) f\left(s, u\left(s, u_{0}\right)\right) d s\right\|_{X^{1+\theta}} \\
\leq & t^{\alpha \theta}\left\|E_{\alpha}\left(t^{\alpha} A\right) u_{0}\right\|_{X^{1+\theta}}+M c \mathbf{B}_{\varepsilon}^{\theta}(\xi) \nu(t) \\
& +M c \mathbf{B}_{\varepsilon}^{\theta}(\xi) \mu^{\rho-1} \sup _{0<s \leq t}\left\{s^{\alpha \varepsilon}\left\|u\left(s, u_{0}\right)\right\|_{X^{1+\varepsilon}}\right\} .
\end{aligned}
$$

Therefore, if $\theta=\varepsilon$ we deduce

$$
\begin{aligned}
t^{\alpha \varepsilon}\left\|u\left(t, u_{0}\right)\right\|_{X^{1+\varepsilon}} \leq t^{\alpha \varepsilon} \| E_{\alpha}\left(t^{\alpha} A\right) & u_{0} \|_{X^{1+\varepsilon}} \\
& +M c \mathbf{B}_{\varepsilon}^{\varepsilon} \nu(t)+\frac{1}{8} \sup _{0<s \leq t}\left\{s^{\alpha \varepsilon}\left\|u\left(s, u_{0}\right)\right\|_{X^{1+\varepsilon}}\right\},
\end{aligned}
$$

from which we obtain

$\sup _{0<s \leq t}\left\{s^{\alpha \varepsilon}\left\|u\left(s, u_{0}\right)\right\|_{X^{1+\varepsilon}}\right\} \leq \frac{8}{7}\left(\sup _{0<s \leq t}\left\{s^{\alpha \varepsilon}\left\|E_{\alpha}\left(s^{\alpha} A\right) u_{0}\right\|_{X^{1+\varepsilon}}\right\}+M c \mathbf{B}_{\varepsilon}^{\varepsilon} \nu(t)\right) \rightarrow 0$

as $t \rightarrow 0$. From above we also conclude that Claim 3 holds.

Claim 4. $\lim _{t \rightarrow 0^{+}}\left\|u\left(t, u_{0}\right)-u_{0}\right\|_{X^{1}}=0$.

For this observe that from Lemma 4.2,

$$
\begin{aligned}
& \left\|u\left(t, u_{0}\right)-u_{0}\right\|_{X^{1}} \\
& \quad \leq\left\|E_{\alpha}\left(t^{\alpha} A\right) u_{0}-u_{0}\right\|_{X^{1}}+\int_{0}^{t} \|(t-s)^{\alpha-1} E_{\alpha, \alpha}\left((t-s)^{\alpha} A\right) f\left(s, u\left(s, u_{0}\right) \|_{X^{1}} d s\right. \\
& \quad \leq\left\|E_{\alpha}\left(t^{\alpha} A\right) u_{0}-u_{0}\right\|_{X^{1}}+M c \mathbf{B}_{\varepsilon}^{0}(\xi)\left(\nu(t)+\left[\sup _{0<s \leq t}\left\{s^{\alpha \varepsilon}\left\|u\left(s, u_{0}\right)\right\|_{X^{1+\varepsilon}}\right\}\right]^{\rho}\right) .
\end{aligned}
$$

Therefore, the claim follows and we have that $u\left(t, u_{0}\right)$ is an $\varepsilon$-regular mild solution starting at $u_{0}$ and it is the unique $\varepsilon$-regular mild solution, starting at $u_{0}$, in the set $K\left(\tau_{0}\right)$. We will call it the $K$-solution starting at $u_{0}$. 
462 B. de Andrade - A.N. Carvalho - P.M. Carvalho-Neto - P. Marín-Rubio

Moreover, if $u_{0}, w_{0} \in B_{X^{1}}\left(v_{0}, r\right)$, it follows from Lemma 4.3 and the choice of $\tau_{0}$ that

$$
\begin{aligned}
t^{\alpha \theta} \| u\left(t, u_{0}\right) & -u\left(t, w_{0}\right)\left\|_{X^{1+\theta}} \leq t^{\alpha \theta}\right\| E_{\alpha}\left(t^{\alpha} A\right)\left(u_{0}-w_{0}\right) \|_{X^{1+\theta}} \\
& +t^{\alpha \theta} \| \int_{0}^{t}(t-s)^{\alpha-1} E_{\alpha, \alpha}\left((t-s)^{\alpha} A\right)\left(f\left(s, u\left(s, u_{0}\right)\right)\right. \\
& -f\left(s, u\left(s, w_{0}\right)\right) d s \|_{X^{1+\theta}} \\
\leq & M\left\|u_{0}-w_{0}\right\|_{X^{1}}+M c \mathbf{B}_{\varepsilon}^{\theta}(\zeta+\alpha \varepsilon) \nu(t) t^{\alpha(\gamma(\varepsilon)-\theta)-\zeta} \\
& \times \sup _{0<s \leq \tau_{0}}\left\{s^{\alpha \theta}\left\|u\left(s, u_{0}\right)-u\left(s, w_{0}\right)\right\|_{X^{1+\varepsilon}}\right\} \\
& +M c \mathbf{B}_{\varepsilon}^{\theta}(\zeta+\alpha \varepsilon) 2 \mu^{\rho-1} t^{\alpha(\gamma(\epsilon)+\varepsilon-\theta-\rho \varepsilon)} \\
& \times \sup _{0<s \leq \tau_{0}}\left\{s^{\alpha \theta}\left\|u\left(s, u_{0}\right)-u\left(s, w_{0}\right)\right\|_{X^{1+\varepsilon}}\right\} .
\end{aligned}
$$

For $\theta=\varepsilon$ we obtain

$$
\begin{aligned}
& t^{\alpha \varepsilon}\left\|u\left(t, u_{0}\right)-u\left(t, w_{0}\right)\right\|_{X^{1+\varepsilon}} \\
& \quad \leq M\left\|u_{0}-w_{0}\right\|_{X^{1}}+\frac{1}{2} \sup _{0<s \leq \tau_{0}}\left\{s^{\alpha \varepsilon}\left\|u\left(t, u_{0}\right)-u\left(t, w_{0}\right)\right\|_{X^{1+\varepsilon}}\right\},
\end{aligned}
$$

which implies

$$
t^{\alpha \varepsilon}\left\|u\left(t, u_{0}\right)-u\left(t, w_{0}\right)\right\|_{X^{1+\varepsilon}} \leq 2 M\left\|u_{0}-w_{0}\right\|_{X^{1}} .
$$

For $0 \leq \theta \leq \theta_{0}<\gamma(\varepsilon)+\varepsilon-\rho \varepsilon$ we have from (4.2) that

$$
\begin{aligned}
& t^{\alpha \theta}\left\|u\left(t, u_{0}\right)-u\left(t, w_{0}\right)\right\|_{X^{1+\theta}} \leq M\left\|u_{0}-w_{0}\right\|_{X^{1}} \\
& \quad+2 M^{2} c \mathbf{B}_{\varepsilon}^{\theta}(\zeta+\alpha \varepsilon)\left[\nu(t) t^{\alpha(\gamma(\varepsilon)-\theta)-\zeta}+2 \mu^{\rho-1} t^{\alpha(\gamma(\varepsilon)+\varepsilon-\theta-\rho \varepsilon)}\right]\left\|u_{0}-w_{0}\right\|_{X^{1}} \\
& \quad \leq C\left(\theta_{0}\right)\left\|u_{0}-w_{0}\right\|_{X^{1}},
\end{aligned}
$$

where

$$
\begin{array}{r}
C\left(\theta_{0}\right)=M\left(1+2 M \sup _{\substack{t \in\left[0, \tau_{0}\right], 0 \leq \theta \leq \theta_{0}}}\left\{c \mathbf { B } _ { \varepsilon } ^ { \theta } ( \zeta + \alpha \varepsilon ) \left[\nu(t) t^{\alpha(\gamma(\varepsilon)-\varepsilon)-\zeta}\right.\right.\right. \\
\left.\left.\left.+2 \mu^{\rho-1} t^{\alpha(\gamma(\varepsilon)+\varepsilon-\theta-\rho \varepsilon)}\right]\right\}\right) .
\end{array}
$$

This concludes the proof of the theorem.

We have the following two consequences of Theorem 1.7. The first one is that if $f$ is independent of time, we obtain the same result. Observe that this is not just by an application of the theorem, since now we do not have a time-dependent class $\mathcal{F}$ any more. However, it is not difficult to readapt its proof. 
Corollary 4.4. With the above notation, assume that $f$ is independent of time and it is an $\varepsilon$-regular map, for some $\varepsilon>0$, relative to the pair $\left(X^{1}, X^{0}\right)$. Then if $v_{0} \in X^{1}$, there exist $r=r\left(v_{0}\right)>0$ and $\tau_{0}=\tau_{0}\left(v_{0}\right)>0$ such that for every $u_{0} \in B_{X^{1}}\left(v_{0}, r\right)$ there is a continuous function $u\left(\cdot, u_{0}\right):\left[0, \tau_{0}\right] \rightarrow X^{1}$ with $u(0)=u_{0}$, which is an $\varepsilon$-regular mild solution to the problem (1.3) starting at $u_{0}$. Furthermore, this solution satisfies

$$
\begin{array}{ll}
u \in C\left(\left(0, \tau_{0}\right] ; X^{1+\theta}\right), & 0 \leq \theta<\gamma(\varepsilon), \\
\lim _{t \rightarrow 0^{+}} t^{\alpha \theta}\left\|u\left(t, u_{0}\right)\right\|_{X^{1+\theta}}=0, & 0<\theta<\gamma(\varepsilon) .
\end{array}
$$

Moreover, for each $\theta_{0}<\gamma(\varepsilon)+\varepsilon-\rho \varepsilon$ there exists a constant $C>0$ such that if $u_{0}, w_{0} \in B_{X^{1}}\left(v_{0}, r\right)$, then

$$
t^{\alpha \theta}\left\|u\left(t, u_{0}\right)-u\left(t, w_{0}\right)\right\|_{X^{1+\theta}} \leq C\left\|u_{0}-w_{0}\right\|_{X^{1}}
$$

for all $t \in\left[0, \tau_{0}\right], 0 \leq \theta \leq \theta_{0}<\gamma(\varepsilon)+\varepsilon-\rho \varepsilon$.

The second consequence of Theorem 1.7 is the following

COROLlary 4.5. Let $f$ be as in Theorem 1.7 and $K$ a relatively compact set in $X^{1}$, then there exists $\tau_{0}=\tau_{0}(K)$ such that the $\varepsilon$-regular solution starting at $u_{0}$ exists in the time interval $\left[0, \tau_{0}\right]$ for any $u_{0} \in K$.

\section{Comparison and positivity results}

5.1. Linear equations. The following result establishes the equivalence between the positivity of the resolvent operator of $A$ and the positivity of the Mittag-Leffler families.

Proposition 5.1. Let $\left(X, \leq_{X}\right)$ be an ordered Banach space and consider $\alpha \in(0,1)$ and $A: D(A) \subset X \rightarrow X$ a sectorial operator. Suppose that there exists $\lambda_{0}>0$ such that $\left(\lambda^{\alpha}-A\right)^{-1}$ is increasing for any $\lambda>\lambda_{0}$. Then, $E_{\alpha}\left(t^{\alpha} A\right)$ is increasing for all $t \geq 0$. Conversely, if $X \ni u_{0} \mapsto E_{\alpha}\left(t^{\alpha} A\right) u_{0} \in C([0, \infty) ; X)$ is increasing for all $t \geq 0$, then $\left(\lambda^{\alpha}-A\right)^{-1}$ is increasing for any $\lambda>0$.

Proof. The case $t=0$ is easily verified.

To prove the case $t>0$, consider $0 \leq_{X} u_{0} \in X$. From Proposition 2.4, write

$$
E_{\alpha}\left(t^{\alpha} A\right) u_{0}=\lim _{n \rightarrow \infty} \frac{1}{n !} \sum_{k=1}^{n+1} b_{k, n+1}^{\alpha}\left(\left(\frac{n}{t}\right)^{\alpha}\left(\left(\frac{n}{t}\right)^{\alpha}-A\right)^{-1}\right)^{k} u_{0}, \quad t>0 .
$$

If $n \in \mathbb{N}$ is such that $n / t>\lambda_{0}$, then

$$
0 \leq\left(\left(\frac{n}{t}\right)^{\alpha}-A\right)^{-1} u_{0}
$$

Since the values $b_{k, n+1}^{\alpha}$, given by (2.4), are positive real numbers and the positive cone is closed, it follows from (5.1) that $0 \leq_{X} E_{\alpha}\left(t^{\alpha} A\right) u_{0}$. 
464 B. de Andrade - A.N. Carvalho - P.M. Carvalho-Neto - P. Marín-Rubio

Conversely, since the integral is a positive operator and

$$
\lambda^{\alpha-1}\left(\lambda^{\alpha}-A\right)^{-1}=\int_{0}^{\infty} e^{-\lambda t} E_{\alpha}\left(t^{\alpha} A\right) d t \quad \text { for all } \lambda>0,
$$

we conclude that $\left(\lambda^{\alpha}-A\right)^{-1}$ is a positive operator whenever $\lambda>0$.

Remark 5.2. (a) Proposition 5.1 is equivalent to the following result: if $0 \leq_{X} u_{0}$, then the solution of the linear homogeneous problem

$$
\left\{\begin{array}{l}
c D_{t}^{\alpha} u(t)=A u(t), \\
u(0)=u_{0} \in X,
\end{array}\right.
$$

verifies $0 \leq_{X} u(t)$ for all $t \geq 0$. Equivalently, if $u_{1} \leq_{X} u_{0}$, then $v(t) \leq_{X} u(t)$ for all $t \geq 0$, where $u$ and $v$ are the corresponding solutions to the problem with initial conditions $u(0)=u_{0}$ and $v(0)=u_{1}$, respectively.

(b) The assumption on the operator $A$ is equivalent to the following positivity result for elliptic problems: for any $\lambda>\lambda_{0}$ and for any $f$ with $0 \leq_{X} f$, the solution to the problem $\lambda^{\alpha} u-A u=f$ satisfies $0 \leq_{X} u$.

A similar result may be stated in terms of the family $\left\{E_{\alpha, \alpha}\left(t^{\alpha} A\right)\right\}_{t \geq 0}$.

Proposition 5.3. Let $\left(X, \leq_{X}\right)$ be an ordered Banach space and consider $\alpha \in(0,1)$ and $A: D(A) \subset X \rightarrow X$ a sectorial operator. Suppose that there exists $\lambda_{0}>0$ such that $\left(\lambda^{\alpha}-A\right)^{-1}$ is increasing for any $\lambda>\lambda_{0}$. Then, $E_{\alpha, \alpha}\left(t^{\alpha} A\right)$ is increasing for all $t \geq 0$. Conversely, if $X \ni u_{0} \mapsto E_{\alpha, \alpha}\left(t^{\alpha} A\right) u_{0} \in C([0, \infty) ; X)$ is increasing for all $t \geq 0$, then $\left(\lambda^{\alpha}-A\right)^{-1}$ is increasing for any $\lambda>0$.

Proof. It suffices to observe that if $u_{0} \in X$, then

$t^{\alpha-1} E_{\alpha, \alpha}\left(t^{\alpha} A\right) u_{0}=\lim _{n \rightarrow \infty} \frac{1}{n !} \sum_{k=1}^{n} \alpha k b_{k, n}^{\alpha}\left(\frac{n}{t}\right)^{\alpha k+1}\left(\left(\frac{n}{t}\right)^{\alpha}-A\right)^{-(k+1)} u_{0}, \quad t \geq 0$.

Now, we may proceed as in the proof of Proposition 5.1. Conversely, we have that

$$
\left(\lambda^{\alpha}-A\right)^{-1}=\int_{0}^{\infty} e^{-\lambda t} t^{\alpha-1} E_{\alpha, \alpha}\left(t^{\alpha} A\right) d t,
$$

for any $\lambda>0$, which concludes the proof.

Corollary 5.4. Let $\left(X, \leq_{X}\right)$ be an ordered Banach space and consider $\alpha \in$ $(0,1)$ and $A: D(A) \subset X \rightarrow X$ a sectorial operator. Suppose that there exists $\lambda_{0}>0$ such that $\left(\lambda^{\alpha}-A\right)^{-1}$ is increasing for any $\lambda>\lambda_{0}$. Let $u_{f}\left(t, u_{0}\right)$ be the local mild solution in $[0, \tau]$, for some $\tau>0$, to the problem

$$
\left\{\begin{array}{l}
c D_{t}^{\alpha} u(t)=A u(t)+f(t), \quad t>0 \\
u(0)=u_{0} \in X
\end{array}\right.
$$

and suppose that $f \in L^{1}(0, \tau ; X)$. Assume that $u_{1} \leq_{X} u_{0}$ and $f_{1}(t) \leq_{X} f_{0}(t)$ for almost every $t \in(0, \tau)$. Then $u_{f_{1}}\left(t, u_{0}\right) \leq_{X} u_{f_{0}}\left(t, u_{0}\right)$ for all $t \in[0, \tau]$. 
In particular, if $0 \leq_{X} u_{0}$ and $0 \leq_{X} f(t)$ for almost every $t \in(0, \tau)$, then $0 \leq_{X} u_{f}\left(t, u_{0}\right)$ for all $t \in[0, \tau]$.

Proof. Observe that the corresponding solutions $(i=0,1)$ are given by

$$
u_{i}(t)=E_{\alpha}\left(t^{\alpha} A\right) u_{i}+\int_{0}^{t}(t-s)^{\alpha-1} E_{\alpha, \alpha}\left((t-s)^{\alpha} A\right) f_{i}(s) d s .
$$

Since $E_{\alpha}\left(t^{\alpha} A\right) u_{1} \leq_{X} E_{\alpha}\left(t^{\alpha} A\right) u_{0}$ and, for any $0<s<t<\tau$,

$$
E_{\alpha, \alpha}\left((t-s)^{\alpha-1} A\right) f_{1}(s) \leq_{X} E_{\alpha, \alpha}\left((t-s)^{\alpha-1} A\right) f_{0}(s),
$$

the result follows from the fact that the integral is a positive operator.

5.2. Semilinear equations. In this paragraph we deals with comparison results and positivity for the semilinear problem (1.1) where $f:[0, \tau) \times X \rightarrow X$ a continuous function, locally lipschitz in the second variable (uniformly with respect to the first variable), and, eventually, bounded.

Proof of Theorem 1.9. Let $T$ be the operator defined by

$$
T(u)(t)=E_{\alpha}\left(t^{\alpha} A\right) u_{0}+\int_{0}^{t}(t-s)^{\alpha-1} E_{\alpha, \alpha}\left((t-s)^{\alpha} A\right) f(s, u(s)) d s .
$$

We proved in Theorem 1.2 that if $\tau \in\left[0, t_{0}\right]$ and $\beta>0$ are small enough, then $T$ is a contraction in $K=\left\{u \in C([0, \tau] ; X):\left\|u(t)-u_{0}\right\| \leq \beta\right\}$ and it has a unique fixed point. Consider $y_{0}(s)=u_{0}, s \in[0, \tau]$. Consequently, $0 \leq_{X} y_{0}(s)$ and $0 \leq_{X} f\left(s, y_{0}(s)\right)$ almost everywehre in $(0, \tau)$. Hence, $y_{1}(t)=T\left(y_{0}(t)\right), t \in[0, \tau]$ satisfies $0 \leq_{X} y_{1}(t)$. Iterating, we obtain

$$
0 \leq_{X} y_{n}(t)=T\left(y_{n-1}(t)\right), \quad t \in[0, \tau] .
$$

Since $\left\{y_{n}\right\}_{n \in \mathbb{N}}$ converges to $u$ in $C([0, \tau] ; X)$ we have that $0 \leq_{X} u(t)$ for all $t \in[0, \tau]$. Now, combining this with some continuation arguments from Section 3 , we may conclude that $0 \leq_{X} u(t)$ for all $t \in\left[0, t_{0}\right]$.

Proof of Theorem 1.10. (a) For $i=0,1$, we know that $u_{i}(t)=u_{f}\left(t, u_{i}\right)$, is a fixed point of the operator

$$
T(u)(t)=E_{\alpha}\left(t^{\alpha} A\right) u_{i}+\int_{0}^{t}(t-s)^{\alpha-1} E_{\alpha, \alpha}\left((t-s)^{\alpha} A\right) f(s, u(s)) d s
$$

in $\left[0, t_{i}\right]$.

Consider initially the function $y_{i}^{0}(t)=u_{i}, t \in\left[0, t_{i}\right], i=0,1$. Iterating, we have $y_{i}^{n}(t)=T\left(y_{i}^{n-1}\right)(t)$ and this sequence converges to $u_{i}(t)$ in $C\left(\left[0, t_{i}\right] ; X\right)$. Furthermore, we have $y_{1}^{0}(t) \leq_{X} y_{0}^{0}(t)$ for almost every $t \in\left(0, t^{*}\right)$ and hence

$$
f\left(t, y_{1}^{0}(t)\right) \leq_{X} f\left(t, y_{0}^{0}(t)\right) \text { for a.e. } t \in\left(0, t^{*}\right) .
$$

Then, $y_{1}^{1}(t) \leq_{X} y_{0}^{1}(t)$ in $\left[0, t^{*}\right]$. Iterating, we obtain $y_{1}^{n}(t) \leq_{X} y_{0}^{n}(t)$ and consequently $u_{1}(t) \leq_{X} u_{0}(t)$ in $\left[0, t^{*}\right]$.

Statements (b) and (c) can be proved analogously. 
466 B. de Andrade - A.N. Carvalho - P.M. Carvalho-Neto - P. Marín-Rubio

Acknowledgments. This paper was started during a P.M.-R.'s visit to the Instituto de Ciências Matemáticas e de Computação (ICMC) at the Universidade de São Paulo, in São Carlos (Brazil), and finished while B.A. and P.M.C.-N. were visiting the Departamento de Ecuaciones Diferenciales y Análisis Numérico (EDAN) at the Universidad de Sevilla in 2011-2012. The authors would like to thanks to the people of these two institutions for their hospitality during their respective stays, and in particular P.M-R. would also like to thank to Ministerio de Educación-DGPU (Spain) for its support, under project PHB2010-0002-PC.

\section{REFERENCES}

[1] H. Amann, Nonhomogeneous linear and quasilinear elliptic and parabolic boundary value problems, Schmeisser/Triebel: Function Spaces, Differential Operators and Nonlinear Analysis, Teubner, Stuttgar, 133 (1993), 9-126.

[2] J.M. Arrieta And A.N. Carvalho, Abstract parabolic problems with critical nonlinearities and applications to Navier-Stokes and Heat equations, Trans. Amer. Math. Soc. 352 (2000), 285-310.

[3] E. Bazhiekova, Fractional Evolution Equations in Banach Spaces, Ph.D. Thesis, Eindhoven University of Technology, 2001.

[4] M.N. Berberan-Santos, Properties of the Mittag-Leffler relaxation function, J. Math. Chem. 38 (2005), 629-635.

[5] H. Brezis and T. Cazenave, A nonlinear heat equation with singular initial data, J. Anal. Math. 68 (1996), 277-304.

[6] A.N. Carvalho and M.R.T. Primo, it Spatial homogeneity in parabolic problems with nonlinear boundary conditions, Commun. Pure Appl. Anal. 3 (2004), 637-651.

[7] S.D. Eidelman And A.N. Kochubei, Cauchy problem for fractional diffusion equations, J. Differential Equations 199 (2004), 211-255.

[8] L. Galeone And R. GarrapPa, Explicit methods for fractional differential equations and their stability properties, J. Comput. Appl. Math. 228 (2009), 548-560.

[9] D. Henry, Geometric Theory of Semilinear Parabolic Equations, Lecture Notes in Mathematics 840, Springer-Verlag, Berlin, 1981.

[10] H.J. Haubold, A.M. Mathai and R.K. Saxena, Mittag-Leffler functions and their applications, J. Appl. Math. 2011 (2011), 51 pages.

[11] A.A. Kilbas, H.M. Srivastava and J.J. Trujillo, Theory and Applications of Fractional Differential Equations, Elsevier, Amsterdam, 2006.

[12] A.A. Kilbas, T. Pierantozzi, J.J. Trujillo and L. Vazquez, On the solution of fractional evolution equations, J. Phys. A Math. Gen. 37 (2004), 3271-3283.

[13] F. Mainard, Fractional calculus and waves in linear viscoelasticity, Imperial College Press, London, 2010.

[14] K.B. Oldham and J. Spanier, The Fractional Calculus, Academic Press, London, 1974.

[15] I.V. OstrovskiI And I.N. Peresyolkova, Nonasymptotic results on distribution of zeros of the function $E_{\rho}(z, \mu)$, Anal. Math. 23 (1997), 283-296.

[16] J. Peng And K. LI, A note on property of the Mittag-Leffler function, J. Math. Anal. Appl. 370 (2010), 635-638.

[17] I. Podlubny, Fractional Differential Equations, Academic Press, San Diego, 1999.

[18] S.G. Samko, A.A. Kilbas and O.I. Marichev, Fractional Integrals and Derivatives. Theory and Applications, Gordon \& Breach Sci. Publishers, Yverdon, 1993. 
[19] W.R. SchneIder, Fractional diffusion, Lecture Notes in Phys. 355 (1990), 276-286.

[20] W.R. SchneIDER AND W. Wyss, Fractional diffusion and wave equations, J. Math. Phys. 30 (1989), 134-144.

[21] R.N. Wang, D.H. Chen And T.J. XiaO, Abstract fractional Cauchy problems with almost sectorial operators, J. Differential Equations 252 (2012), 202-235.

[22] R.N. WANG, T.J. XIAO AND J. LiAng, A note on the fractional Cauchy problems with nonlocal initial conditions, Appl. Math. Lett. 24 (2011), 1435-1442.

[23] F.B. Weissler, Semilinear evolution equations in Banach spaces, J. Funct. Anal. 32 (1979), 277-296.

[24] _ Local existence and nonexistence for semilinear parabolic equations in $L^{p}$, Indiana Univ. Math. J. 29 (1980), 79-102.

[25] W. Wyss, The fractional diffusion equation, J. Math. Phys. 27 (1986), 2782-2785.

[26] K. Yosida, Functional Analysis, Springer-Verlag, New York, 1978.

Manuscript received Fabruary 16, 2013

Bruno de Andrade, Alexandre N. Carvalho and Paulo M. Carvalho-Neto

Instituto de Ciências Matemáticas e de Computação

Universidade de São Paul

Campus de São Carlos

Caixa Postal 668

13560-970 São Carlos SP, BRAZIL

E-mail address: bruno00luis@gmail.com, andcarva@icmc.usp.br, pmat@icmc.usp.br

Pedro Marín-Rubio

Departamento de Ecuaciones Diferenciales y Análisis Numérico

Universidad de Sevilla

Apdo. de Correos 1160

41080-Sevilla, SPAIN

E-mail address: pmr@us.es 